\title{
FUN3D Analyses in Support of the Second Aeroelastic Prediction Workshop
}

\author{
Pawel Chwalowski, ${ }^{*}$ Jennifer Heeg ${ }^{\dagger}$ \\ NASA Langley Research Center, Hampton, VA 23681-2199
}

\begin{abstract}
This paper presents the computational aeroelastic results generated in support of the second Aeroelastic Prediction Workshop for the Benchmark Supercritical Wing (BSCW) configurations and compares them to the experimental data. The computational results are obtained using FUN3D, an unstructured grid ReynoldsAveraged Navier-Stokes solver developed at NASA Langley Research Center. The analysis results include aerodynamic coefficients and surface pressures obtained for steady-state, static aeroelastic equilibrium, and unsteady flow due to a pitching wing or flutter prediction. Frequency response functions of the pressure coefficients with respect to the angular displacement are computed and compared with the experimental data. The effects of spatial and temporal convergence on the computational results are examined.
\end{abstract}

\section{Nomenclature}

\section{Roman Symbols}

$C_{p} \quad$ Coefficient of pressure

M, Mach Mach number

$q \quad$ Dynamic pressure

Greek Symbols

$\alpha, \theta \quad$ Angle of attack, angular displacement

$f, f^{*} \quad$ frequency, system's primary frequency - $\mathrm{Hz}$

Acronyms

AePW Aeroelastic Prediction Workshop

BSCW Benchmark Supercritical Wing

CAE Computational Aeroelasticity

CFD Computational Fluid Dynamics

CSD Cross Spectral Density

DDES Delayed Detached Eddy Simulation

DFT Discrete Fourier Transform

DPW Drag Prediction Workshop

FRF Frequency Response Function

HiLiftPW High Lift Prediction Workshop

OTT Oscillating Turntable

PAPA Pitch And Plunge Apparatus

PSD Power Spectral Density

${ }^{*}$ Senior Aerospace Engineer, Aeroelasticity Branch, MS 340, Senior Member AIAA.

${ }^{\dagger}$ Senior Research Engineer, Aeroelasticity Branch, MS 340, Senior Member AIAA. 


\section{Introduction}

$\mathrm{T}$ he first Aeroelastic Prediction Workshop (AePW-1) was held in April 2012 1 -3 and served as a first step in assessing the state-of-the-art of computational methods for predicting unsteady flow fields and aeroelastic response. The second Aeroelastic Prediction Workshop (AePW-2), held in January 2016, built on the experiences of the first workshop by extending the benchmarking effort to aeroelastic flutter solutions. The configuration chosen for the AePW-2 was the Benchmark Supercritical Wing (BSCW). ${ }^{4}$

The accuracy of computational methods has improved in recent years due to individual validation efforts like those with the AGARD 445.6 wing, ${ }^{5}$ but multi-analyst code-to-code comparisons that can be used to assess the overall current state-of-the-art in computational aeroelasticity (CAE) are limited. The idea for an Aeroelastic Prediction Workshop (AePW) series was conceived at NASA in 2009, based on the success of two other workshop series that have been conducted over the past decade: the Drag Prediction Workshop (DPW) ${ }^{6}$ series and the High Lift Prediction Workshop (HiLiftPW) ${ }^{7}$ series. The intent for the AePW was to provide a forum for code-to-code comparisons involving predictions of nonlinear aeroelastic phenomena and to stimulate upgrades for existing codes and the development of new codes.

For code validations in general, the type of aerodynamic and/or aeroelastic phenomena to be analyzed is important since a validation process typically progresses from simpler to more challenging cases. For the AePW series, the approach being taken is to utilize existing experimental data sets in a building-block approach to incrementally validate targeted aspects of CAE tools. Each block will represent a component of a more complex nonlinear unsteady aeroelastic problem, isolating it such that the contributing physics can be thoroughly investigated. The challenge selected for the first AePW was the accurate prediction of unsteady aerodynamic phenomena on essentially rigid, geometrically simple models, with an additional foray into systems with weak coupling between the fluid and the structure. Results from this first workshop helped guide the direction of the second workshop, with analyses extending to include flutter prediction and therefore, increasingly complicated flow fields.

This paper presents the computational aeroelastic results generated for the BSCW configuration using the NASA Langley-developed computational fluid dynamics (CFD) software FUN3D ${ }^{8}$ and comparing those results to the experimental data. Information relevant to the numerical method employed will be presented first, including the grids used, the rigid steady-flow analysis, the dynamic analysis, and the post processing. Details associated with the BSCW test cases and their associated analyses using FUN3D will then be presented. Due to the large number of computational analyses and data generated, this paper presents only a subset of the results obtained for each test case.

\section{Numerical Method}

\section{A. Grids}

Unstructured grids consisting primarily of tetrahedra and prisms were used in this study. They were generated using VGRID $^{9}$ with input prepared using GridTool. ${ }^{10}$ The tetrahedral elements within the boundary layer were converted into prism elements using preprocessing options within the FUN3D software. Based on the AePW gridding guidelines, ${ }^{11}$ three grids belonging to the same family were constructed. These grids and the corresponding FUN3D solutions are referred to as 'coarse', 'medium' and 'fine' in this paper. In addition, a separate and preliminary grid was constructed for use with the Delayed Detached Eddy Simulation (DDES) method. The resulting grid distributions for the surface and plane of symmetry are presented in Figure 1.

\section{B. Rigid Steady-Flow Analysis - FUN3D}

Solutions to the Reynolds-Averaged Navier-Stokes (RANS) equations were computed using the FUN3D flow solver with turbulence closure obtained using the "standard" Spalart-Allmaras one-equation model. ${ }^{12,13}$ For the test cases at the low transonic Mach numbers selected for the BSCW, oscillations in pressure fore and aft of the shock are relatively minor, so that the use of a flux limiter, with the accompanying adverse effects on solver convergence is not essential; however, selected analyses were completed with and without a flux limiter. Here, the flux limitation was accomplished with the Venkatakrishnan ${ }^{14}$ limiter, and inviscid fluxes were computed using the Roe scheme. ${ }^{15}$ For the asymptotically-steady cases, time integration was accomplished by an Euler implicit backwards difference scheme, with local time stepping to accelerate convergence. Most of the cases in this study were run for about 5000 iterations to achieve convergence of forces and moments to within $\pm 0.5 \%$ of the running average of the last 1,000 iterations. 


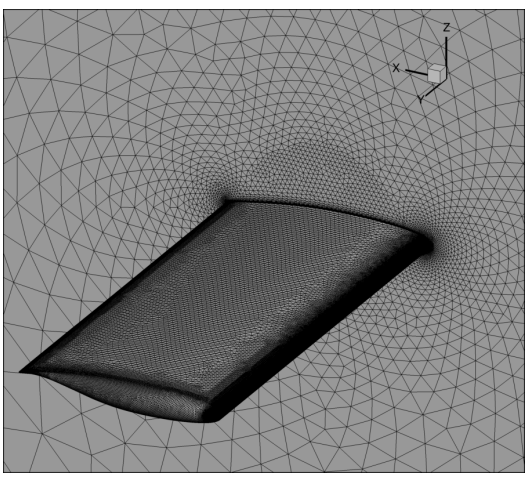

(a) Coarse Grid, Grid A, 3 Million Nodes.

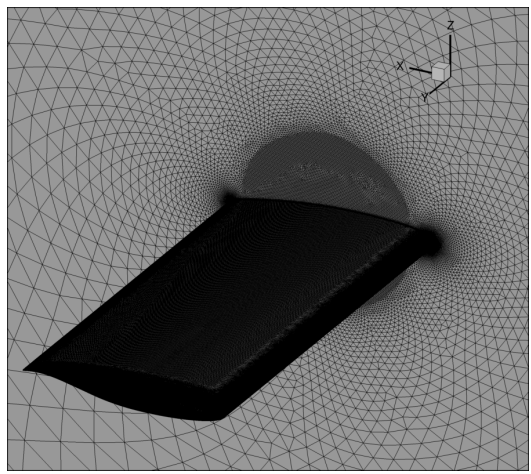

(c) Fine Grid, Grid C, 27 Million Nodes.

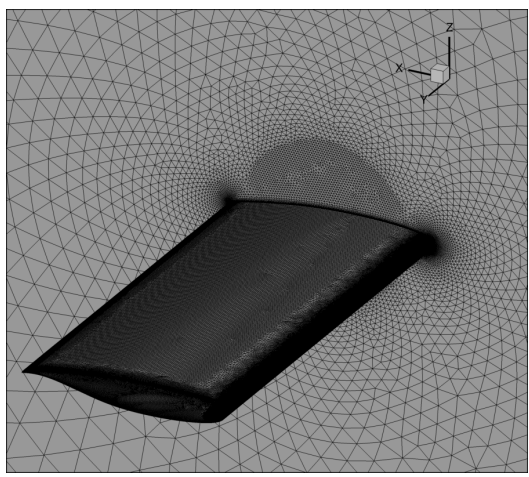

(b) Medium Grid, Grid B, 9 Million Nodes.

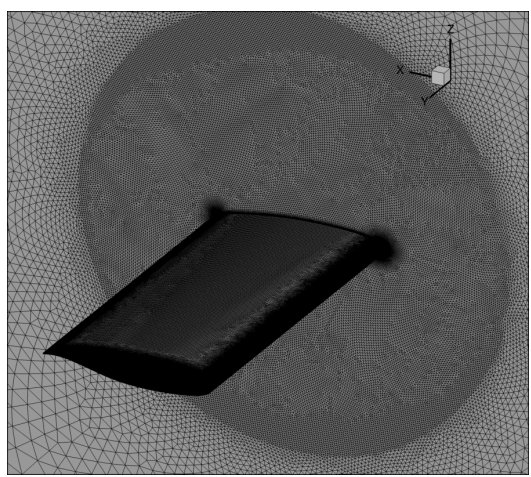

(d) Towards DDES, Grid D, 35 Million Nodes.

Figure 1. Coarse, Medium, Fine, and “Towards DDES” Grids.

\section{Dynamic Analysis - FUN3D}

Dynamic analyses of the BSCW configuration required moving body, and therefore, grid motion capability. The grid deformation in FUN3D is treated as a linear elasticity problem. In this approach, the grid points near the body can move significantly, while the points farther away may not move at all. In addition to the moving body capability, the analysis of the BSCW configuration required dynamic aeroelastic capability, which is available in the FUN3D solver. ${ }^{16}$ For a dynamics aeroelastic analysis, FUN3D is capable of being loosely coupled with an external finite element solver, ${ }^{17}$ or in the case of the linear structural dynamics used in this study, an internal modal structural solver can be utilized. This modal solver is formulated and implemented in FUN3D in a manner similar to other Langley aeroelastic codes $\left(\mathrm{CAP}-\mathrm{TSD}^{18}\right.$ and CFL3D ${ }^{19}$ ). For the BSCW computation presented here, the structural modes were obtained via a normal modes analysis (solution 103) with the Finite Element Model (FEM) solver MSC Nastran ${ }^{T M} .{ }^{20}$ The modes were then interpolated to the surface mesh using the method developed by Samareh. ${ }^{21}$ The BSCW FEM was built and described by Heeg. ${ }^{4}$

The BSCW dynamic analysis was performed in a multi-step process. First, the steady CFD solution was obtained on the rigid body. For the forced-oscillation cases, the BSCW wing was then moved sinusoidally about the pitch axis specified in the experiment. In the case of a dynamic aeroelastic flutter solution, a static aeroelastic solution was obtained first by restarting the CFD analysis from the rigid-steady solution in a time-accurate mode ${ }^{22}$ with a structural modal solver, allowing the structure to deform. A high value of structural damping (0.99) was used so the structure could find its equilibrium position with respect to the mean flow before the dynamic response was started. Finally, the flutter solution was restarted from the static aeroelastic solution by setting the structural damping value to zero and reducing the time step to approximately 500 or 5000 time steps per cycle which corresponds to 0.0002 or 0.00002 seconds, respectively. A detailed temporal resolution study was also conducted and will be documented in future publications; however, some aspects of the temporal resolution issues that were identified during this study are described later in this paper. 


\section{Post Processing}

The dynamic comparison data selected for AePW-2 consisted of the magnitudes and phases of frequency response functions (FRFs). The FRFs of principal interest were the pressure coefficients $\left(\mathrm{C}_{p}\right)$ due to angular displacement. The FRF for each pressure coefficient was calculated at the principal frequency of the reference quantity (angular displacement).

Fourier analysis was performed on each dynamically-excited data set to produce FRFs for each pressure relative to the displacement of the system. The FRFs were formed from power spectral and cross spectral densities (PSDs and CSDs), which were computed using Welch's periodogram method. The Fourier coefficients used in computing the PSDs and CSDs were generated using discrete Fourier transform (DFT) analysis of the time histories, employing overlap-averaged ensembles of the data sets. The length of the ensembles and the frequency at which the data was extracted were chosen based on statistical analysis of the results of varying the ensemble lengths. The objective in varying the block size was to exactly match the system's primary frequency with a Fourier analysis frequency and then maximize the number of data blocks to reduce the processing-based uncertainty. A rectangular windowing was used; the windows were overlapped by $90 \%$ of the block size.

For BSCW, the magnitude of the frequency response function of $\mathrm{C}_{p}$ due to $\theta$ at the frequency $f(i)$ as a function of chord location is presented in this paper as:

$$
\left|\frac{C_{p}}{\theta}\left(f^{*}\right)\right| v s \cdot \frac{x}{c}
$$

where $f^{*}$ represents the system's primary frequency.

\section{AePW-2 Test Cases}

The BSCW model, shown in Figure 2, has a simple, rectangular, 16- $\mathrm{x}$ 32-inch wing planform, with a NASA SC(2)-0414 airfoil. The BSCW geometric reference parameters are shown in Table 1. The model was mounted to a large splitter plate, sufficiently offset from the wind-tunnel wall (40 inches) to (1) place the wing closer to the tunnel centerline and (2) be outside the tunnel wall boundary layer. ${ }^{23}$ The wing was designed to be rigid, with the following structural frequencies for the combined installed wing and OTT mounting system: $24.1 \mathrm{~Hz}$ (spanwise first bending mode), $27.0 \mathrm{~Hz}$ (in-plane first bending mode), and $79.9 \mathrm{~Hz}$ (first torsion mode). For instrumentation, the model has pressure ports at two chord-wise rows at the $60 \%$ and $95 \%$ span locations, with 22 ports on the upper surface, 17 ports on the lower surface, and 1 port at the leading edge for each row. The BSCW model was tested in the NASA Transonic Dynamics Tunnel (TDT) in two test entries. Differences between the two tests and their associated data sets are provided in Table 2. The first BSCW test in the early 1990's, was performed on a flexible mount system, called the pitch and plunge apparatus (PAPA), which provides two-degree-of-freedom low-frequency flexible modes that emulate a plunge mode and a pitch mode. The BSCW/PAPA data consists of unsteady data at flutter points and averaged data on a rigidified apparatus at the flutter conditions. For this PAPA test, both the inboard row at the $60 \%$ span station and the outboard row at the $95 \%$ span station were populated with unsteady in-situ pressure transducers. The more recent test in 2000, which served as the basis for one of the AePW-1 test cases, was performed on the oscillating turntable (OTT), which provided forced pitch oscillation data. For this OTT test, however, only the inboard row at the $60 \%$ span station was populated with unsteady in-situ pressure transducers.

Both of the TDT tests were conducted with the sidewall slots open. The BSCW/PAPA test was conducted with several flow transition strip configurations, but only data using size \#35 grit was used for the workshop comparisons. For the BSCW/OTT test, the boundary-layer transition was also fixed at $7.5 \%$ chord using size \#30 grit.

The BSCW flow conditions used in AePW-1 were challenging. Shock-induced separated flow dominated the upper surface and the aft portion of the lower surface at the Mach 0.85 and $5^{\circ}$ angle-of-attack case. Using this information as a guide, two test cases just outside of the separated flow regime were emphasized for AePW-2 and are listed in Table 3. Steady and forced oscillation analyses were conducted at Mach $0.7,3^{\circ}$ angle of attack, and flutter analyses were conducted at Mach $0.74,0^{\circ}$ angle of attack. An optional Case \#3 at Mach $0.85,5^{\circ}$ angle of attack, which was the re-analysis of the AePW-1 case, was encouraged to apply the higher fidelity tools. This optional case was divided into three separate sub-cases based on the type of dynamic data acquired.

FUN3D analyses were conducted for all AePW-2 cases listed in Table 3. In the following sections of this paper, some of the FUN3D computational results obtained for the AePW-2 BSCW test cases will be presented with the corresponding experimental data. 


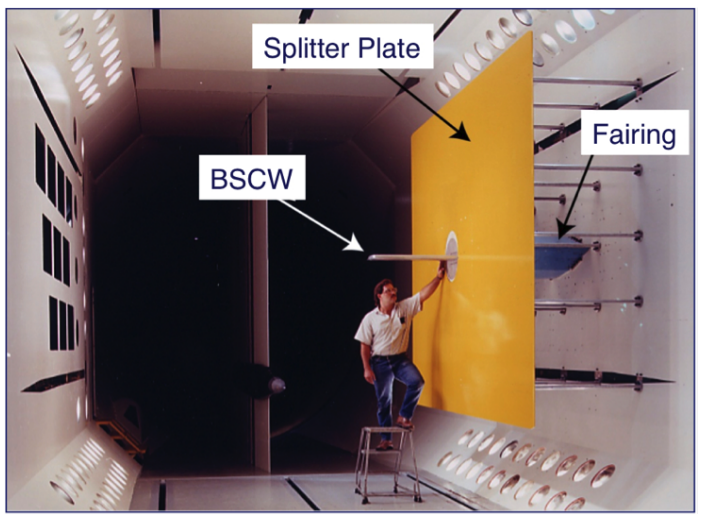

(a) Photograph of the BSCW model mounted on the OTT in the TDT.

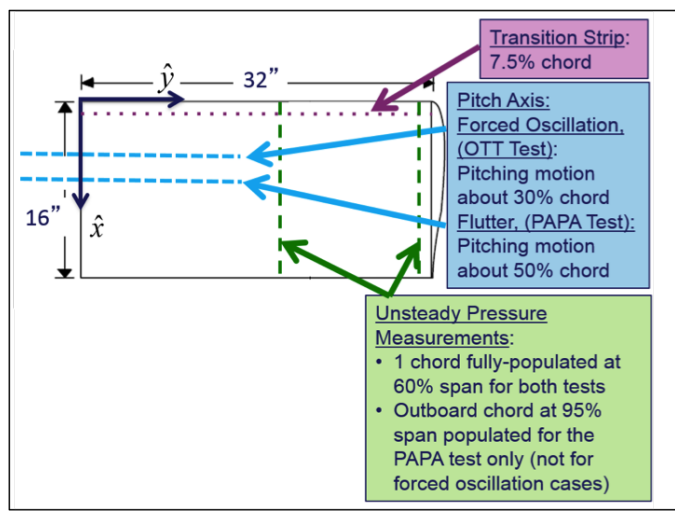

(b) BSCW geometry.

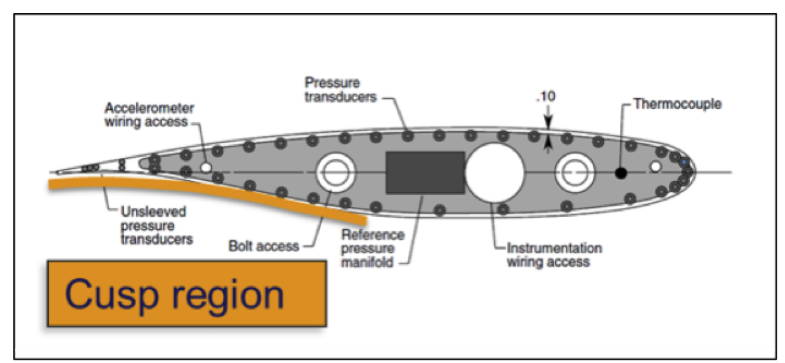

(c) Cross-sectional view of the SC(2)-0414 airfoil, with BSCW instrumentation.

Figure 2. BSCW Model.

Table 1. BSCW Geometric Reference Parameters.

\begin{tabular}{l|c|c}
\hline \hline Description & Symbol & Value \\
\hline Reference chord & $c_{\text {ref }}$ & 16 inches \\
\hline Model span & $b$ & 32 inches \\
\hline Area & $A$ & 512 inch $^{2}$ \\
\hline Moment reference point & $x_{\text {ref }}$ & 4.8 inches, $30 \%$ \\
relative to axis system def. & $y_{\text {ref }}$ & 0.0 inches \\
& $z_{\text {ref }}$ & 0.0 inches \\
\hline Frequency Response Function & & \\
reference quantity & FRF & Pitch angle \\
\hline \hline
\end{tabular}

Table 2. Differences between the Two BSCW TDT Test Configurations and Associated Data Sets.

\begin{tabular}{c|c|c}
\hline \hline Test number & 470 (year 1991) & 548 (year 2000) \\
\hline Mount system & PAPA & OTT \\
\hline Pitch axis, \% chord & $50 \%$ & $30 \%$ \\
\hline Test medium & R-12 & R-134a \\
\hline Pressure transducer spanwise locations & $60 \%, 95 \%$ & $60 \%$ \\
\hline Steady data configuration & Rigidized mount system & Unforced system \\
\hline Forced oscillation data? & No & Yes \\
\hline Flutter data? & Yes & No \\
\hline Time history records? & No & Yes \\
\hline \hline
\end{tabular}


Table 3. AePW-2 Workshop Test Cases.

\begin{tabular}{l|c|c|c|c|c}
\hline \hline & Case \#1 & Case \#2 & Optional Case \#3a & Optional Case \#3b & Optional Case \#3c \\
\hline Mach & 0.7 & 0.74 & 0.85 & 0.85 & 0.85 \\
\hline AoA & $3^{\circ}$ & $0^{\circ}$ & $5^{\circ}$ & $5^{\circ}$ & $5^{\circ}$ \\
\hline Dynamic & Forced & Flutter & Unforced & Forced & Flutter \\
Data Type & Oscillation & & Unsteady & $f=10 \mathrm{~Hz},|\theta|=1^{\circ}$ & \\
& $f=10 \mathrm{~Hz},|\theta|=1^{\circ}$ & & & - Separated flow & - Separated flow \\
& - Attached flow & - Flow state(?) & - Separated flow & - OTT exp. data & - No exp. data \\
& Notes: OTT exp. data & - PAPA exp. data & - OTT exp. data & - R-134a & - R-134a \\
& - R-134a & - R-12 & - R-134a & \multicolumn{5}{c}{} \\
\hline \hline
\end{tabular}

\section{A. Test Case \#1}

Steady and forced-oscillation analyses were conducted for the first AePW-2 test case: Mach $0.7,3^{\circ}$ angle of attack. The computed aerodynamic coefficients, including total coefficients and sectional coefficients at the $60 \%$ and $95 \%$ span stations, for the coarse, medium, and fine grids are presented in Table 4. Figures 3(a)-(f) show the mean $C_{p}$ and frequency response functions (FRFs) at the $10 \mathrm{~Hz}$ pitching frequency at the $60 \%$ span station. Figures $3(\mathrm{a})$ and (b) show the computed and experimental mean pressure coefficients on the upper and lower wing surfaces, respectively. There is very good agreement in pressure with the exception of the peak value near the leading edge on the upper surface. This pressure jump in the experimental data suggests that the mean value of $C_{p}$ might not be the best quantity to represent the dynamic behavior of pressure near stagnation point and/or shock. Figures 3(c) and (d) show the magnitude of the FRF of pressure due to angular displacement for the upper and lower surfaces, and Figures 3(e) and (f) show the corresponding phase of the FRF. The upper surface phase plot, Figure 3(e), shows some mismatch in the experimental and computational data past the axis of rotation. While the experimental data shows the pressure to be $-140^{\circ}$ out of phase with pitch angle at chord station $\mathrm{x} / \mathrm{c}=0.85$, the computational data predicts the pressure to be $-60^{\circ}$ out of phase at chord station $\mathrm{x} / \mathrm{c}=0.95$. The reason for that mismatch is difficult to explain. Perhaps the application of a different turbulence model is required, or in general, the grid resolution in the aft region of the wing and in the wake may not be adequate to capture all the flow physics even at this relatively low Mach number and angle of attack. A similar, but not as severe, difference is observed over the aft portion of the lower surface.

Table 4. BSCW Analysis Aerodynamic Coefficients from Steady Rigid Solution, Mach $0.7,3^{\circ}$ Angle of Attack.

\begin{tabular}{c|c|c|c|c|c|c|c|c|c}
\hline \hline Grid & $C_{L}$ & $C_{D}$ & $C_{M y}$ & $C_{l 60}$ & $C_{d 60}$ & $C_{m y 60}$ & $C_{l 95}$ & $C_{d 95}$ & $C_{m y 95}$ \\
\hline Coarse & 0.4354 & 0.0272 & -0.0576 & 0.4438 & 0.0268 & -0.0599 & 0.2344 & 0.0277 & -0.0414 \\
Medium & 0.4371 & 0.0268 & -0.0580 & 0.4452 & 0.0263 & -0.0601 & 0.2349 & 0.0270 & -0.0416 \\
Fine & 0.4311 & 0.0262 & -0.0564 & 0.4393 & 0.0257 & -0.0585 & 0.2327 & 0.0266 & -0.0410 \\
\hline \hline
\end{tabular}

\section{B. Test Case \#2}

The second AePW-2 test case involves BSCW flutter prediction at Mach 0.74 and $0^{\circ}$ angle of attack. The computational process to calculate flutter dynamic pressure and the corresponding frequency requires multiple steps. First, the rigid steady solution is obtained, which is followed by the static aeroelastic solution. The third step is to perform several dynamic aeroelastic FUN3D computations with different values of the dynamic pressure (q) in the vicinity of the experimental flutter dynamic pressure. The wing response in the form of the time varying pitch angle is then computed and used to calculate the damping ratio using the logarithmic decrement method. For the stable solution, the damping ratio is greater than zero, and for the unstable solution, the damping ratio is less than zero. The damping ratio and the dynamic pressure are interpolated, and at zero damping ratio, the dynamic pressure is considered to be the flutter dynamic pressure. Once this flutter dynamic pressure is identified, the corresponding flutter frequency is determined via an interpolation of the frequencies at that condition. In our analysis process, we obtained dynamic solutions for the medium grid at several dynamic pressures, ranging from 1 to 168.8 psf. The computed damping and frequencies are presented in Figure 4 (a) and (b), respectively. A dynamic pressure of 151.2 psf corresponds to 


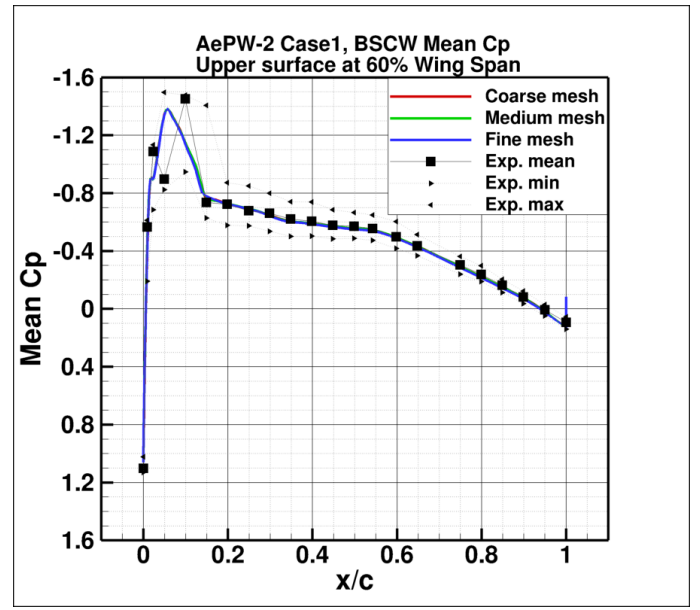

(a) Mean $C_{p}$, Upper Surface.

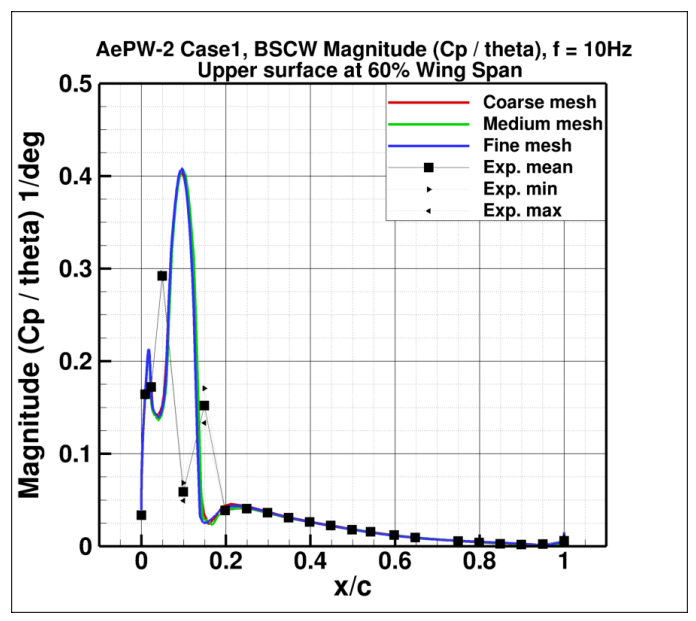

(c) $\mid C_{p}$ /theta $\mid$, Upper Surface.

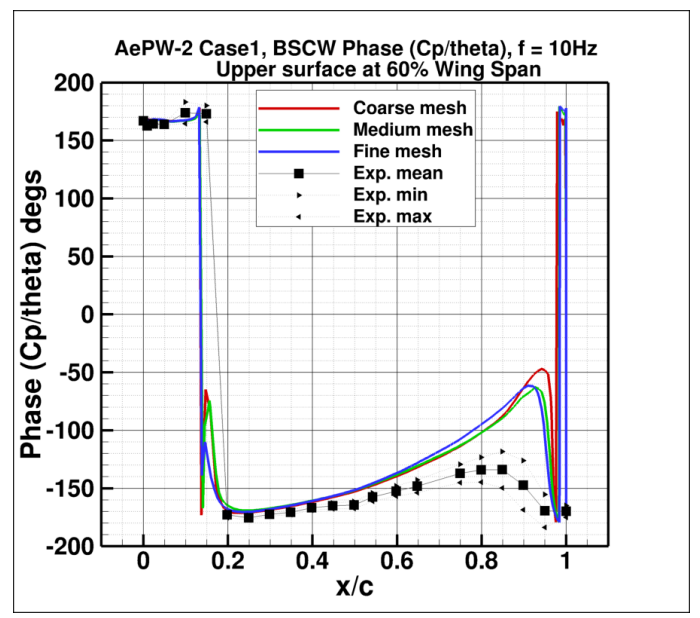

(e) Phase $C_{p} /$ theta, Upper Surface.

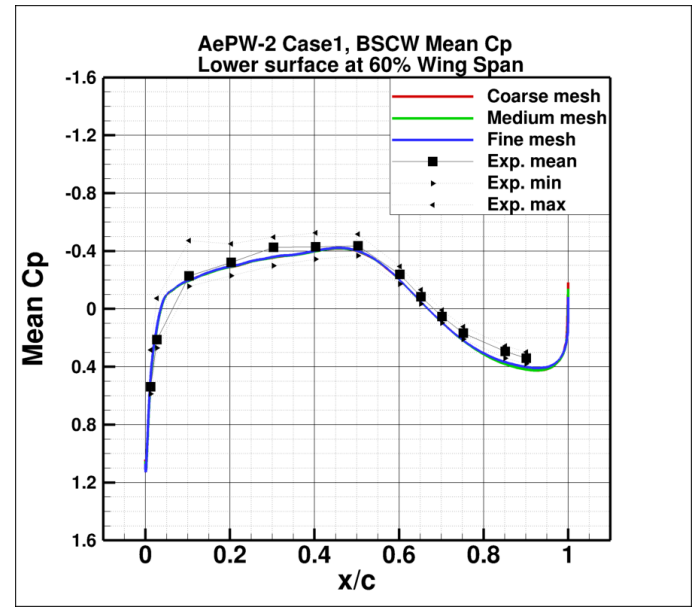

(b) Mean $C_{p}$, Lower Surface.

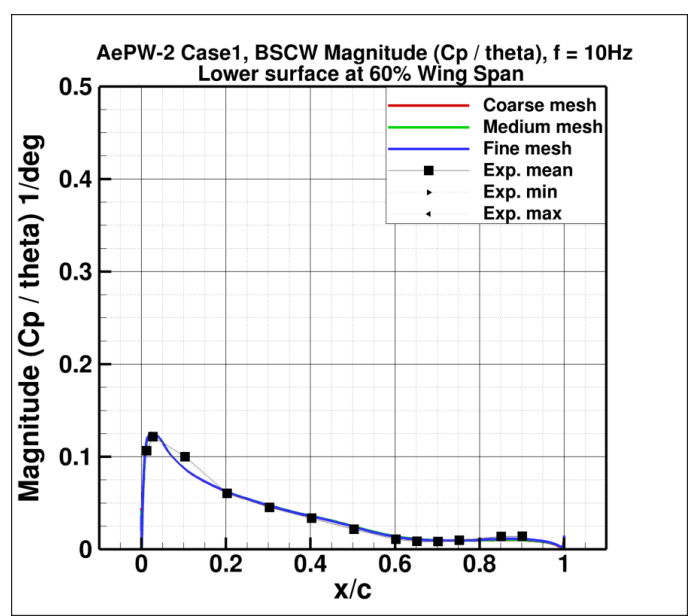

(d) $\mid C_{p} /$ theta $\mid$, Lower Surface.

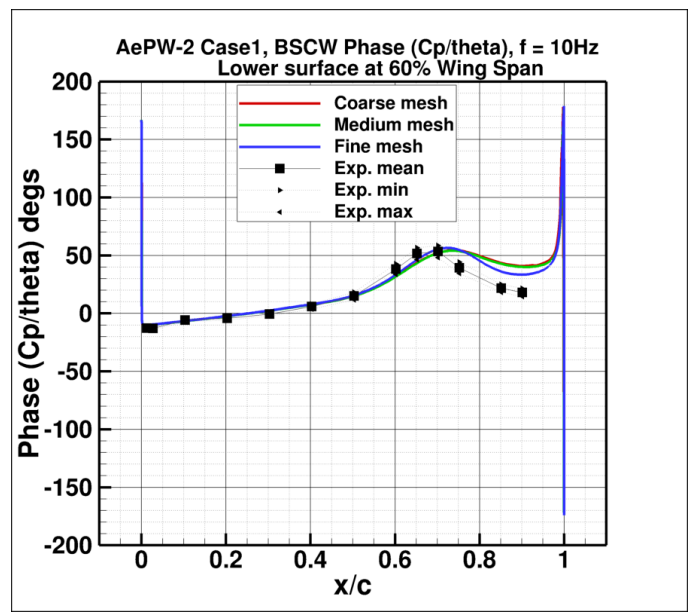

(f) Phase $C_{p} /$ theta, Lower Surface.

Figure 3. Mean $C_{p}$ and Frequency Response Function: Magnitude and Phase of Pressure Due to Pitch Angle, Mach 0.7, Pitching Frequency $f=10 \mathrm{~Hz}$ case, $R e_{c}=4.56 * 10^{6}, \alpha=3^{\circ}$. 
zero damping for the medium grid solution. Based on this medium grid solution, coarse and fine grid solutions were obtained at dynamic pressures of both $152.0 \mathrm{psf}$ and $168.8 \mathrm{psf}$. The resulting coarse and fine grid results predicted the zero damping value to occur at dynamic pressures of $152.5 \mathrm{psf}$ and $151.5 \mathrm{psf}$, respectively. The corresponding flutter frequency for all three grids was then determined to be approximately $4.2 \mathrm{~Hz}$. An averaged dynamic pressure of 151.7 was declared to be the computationally-obtained flutter dynamic pressure while the dynamic pressure of 168.8 psi is an experimentally-measured flutter dynamic pressure. This also means that the computationally-obtained flutter dynamic pressure is approximately $10 \%$ below the experimental flutter dynamic pressure.

Tables 5 and 6 show aerodynamic coefficients obtained from the rigid steady and static aeroelastic solutions, respectively, at the experimental flutter dynamic pressure of $168.8 \mathrm{psf}$. There is a significant lift coefficient reduction between the two solutions. This reduction indicates that the wing pitches down due to the static aeroelastic effects. The predicted pitch-down angle values are $-1.2^{\circ},-1.3^{\circ}$, and $-1.0^{\circ}$ for the coarse, medium, and fine grids, respectively.

Figures 5(a)-(f) and Figures 6(a)-(f) show the mean $C_{p}$ and frequency response functions computed from the FUN3D solution at the experimental flutter dynamic pressure of 168.8 psf at the $60 \%$ and $95 \%$ wing span stations, respectively. Figures 7(a)-(f) and Figures 8(a)-(f) shows the mean $C_{p}$ and frequency response functions computed from the FUN3D solution at the dynamic pressure of $152.0 \mathrm{psf}$ at $60 \%$ and $95 \%$ wing span stations, respectively. There is a very good agreement between the experimental and computational data at both the 152.0 and $168.8 \mathrm{psf}$ dynamic pressures. The largest differences are noted near the leading edge of the wing on the lower surface for both span stations. The other region of noticeable disagreement is in the lower surface cusp region, where the computations predict approximately half the magnitude of response relative to the experiment. At the cusp leading edge, the computational results track the phase change observed in the experiment. Note, that the experimental flutter dynamic pressure is at $q$ $=168.8 \mathrm{psf}$, but for comparison purposes, it is also plotted together with the computational data at $\mathrm{q}=152 \mathrm{psf}$.

Table 5. BSCW Analysis Aerodynamic Coefficients from Steady Rigid Solution, Mach 0.74, $\mathrm{q}=168.8 \mathrm{psf}, \mathrm{AoA}=0^{\circ}$.

\begin{tabular}{c|c|c|c|c|c|c|c|c|c}
\hline \hline Grid & $C_{L}$ & $C_{D}$ & $C_{M y}$ & $C_{l 60}$ & $C_{d 60}$ & $C_{m y 60}$ & $C_{l 95}$ & $C_{d 95}$ & $C_{m y 95}$ \\
\hline Coarse & 0.1840 & 0.0143 & -0.0783 & 0.1905 & 0.0145 & -0.0821 & 0.1030 & 0.0155 & -0.0536 \\
Medium & 0.1843 & 0.0138 & -0.0783 & 0.1906 & 0.0140 & -0.0821 & 0.1018 & 0.0150 & -0.0529 \\
Fine & 0.1762 & 0.0133 & -0.0755 & 0.1825 & 0.0135 & -0.0792 & 0.0982 & 0.0146 & -0.0515 \\
\hline \hline
\end{tabular}

Table 6. BSCW Analysis Aerodynamic Coefficients from Static Aeroelastic Solution, $q=168.8$ psf, $\operatorname{Mach} 0.74, A o A=0^{\circ}$.

\begin{tabular}{c|c|c|c|c|c|c|c|c|c}
\hline \hline Grid & $C_{L}$ & $C_{D}$ & $C_{M y}$ & $C_{l 60}$ & $C_{d 60}$ & $C_{m y 60}$ & $C_{l 95}$ & $C_{d 95}$ & $C_{m y 95}$ \\
\hline Coarse & 0.0935 & 0.0123 & -0.0856 & 0.0992 & 0.0124 & -0.0898 & 0.0601 & 0.0135 & -0.0600 \\
Medium & 0.0937 & 0.0117 & -0.0855 & 0.0992 & 0.0119 & -0.0897 & 0.0583 & 0.0129 & -0.0590 \\
Fine & 0.0890 & 0.0113 & -0.0822 & 0.0946 & 0.0114 & -0.0863 & 0.0556 & 0.0126 & -0.0568 \\
\hline \hline
\end{tabular}

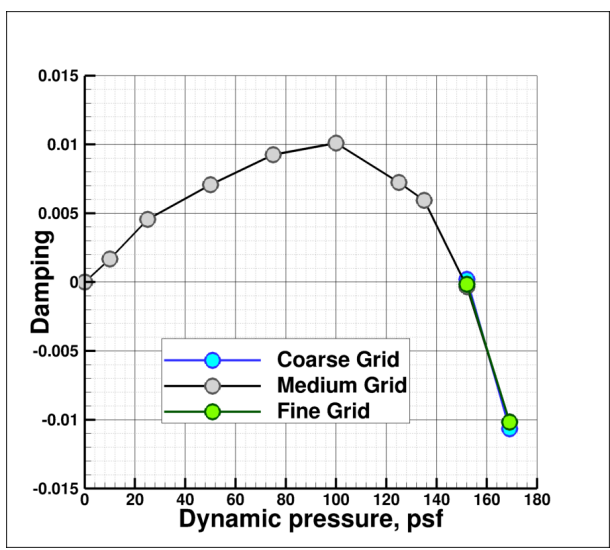

(a) Damping Value as a Function of Dynamic Pressure.

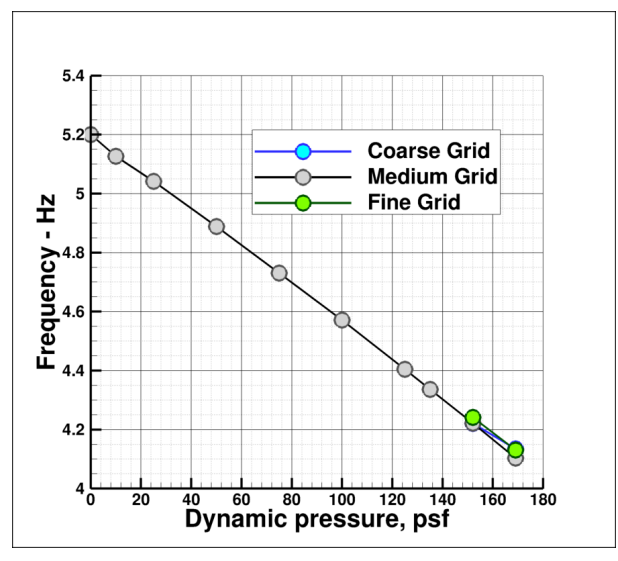

(b) Frequency as a Funtion of Dynamic Pressure.

Figure 4. Case \#2: Search for Flutter Dynamic Pressure and Flutter Frequency, Mach 0.74, $\operatorname{Re}_{c}=4.45 * 10^{6}, \alpha=0^{\circ}$. 


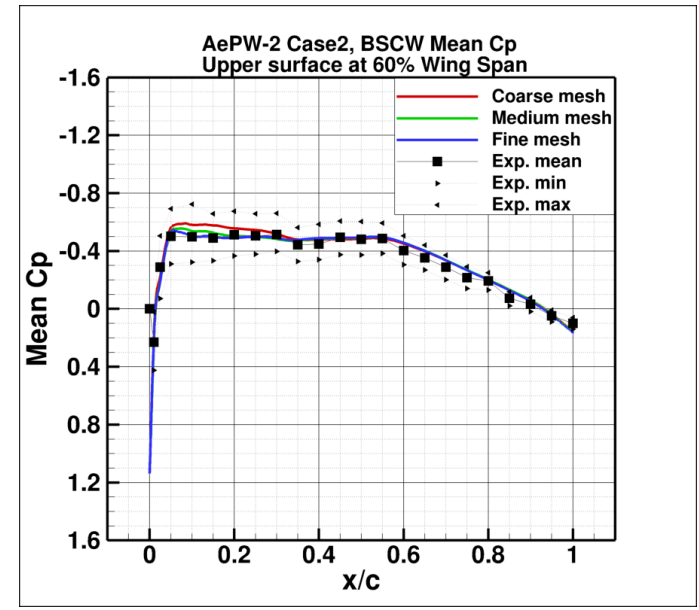

(a) Mean $C_{p}$, Upper Surface.

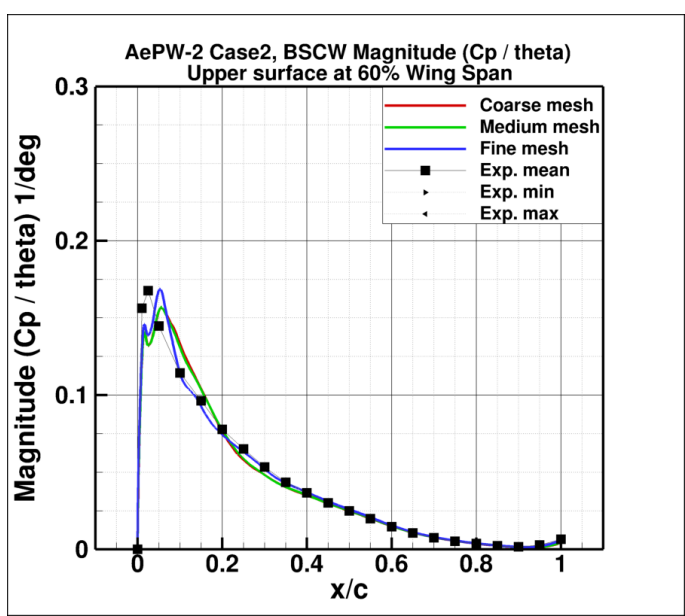

(c) $\mid C_{p} /$ theta $\mid$, Upper Surface.

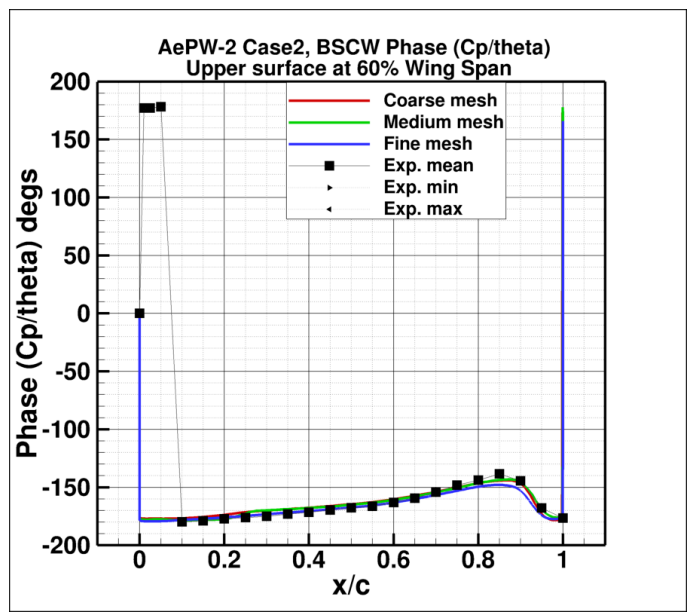

(e) Phase $C_{p} /$ theta, Upper Surface.

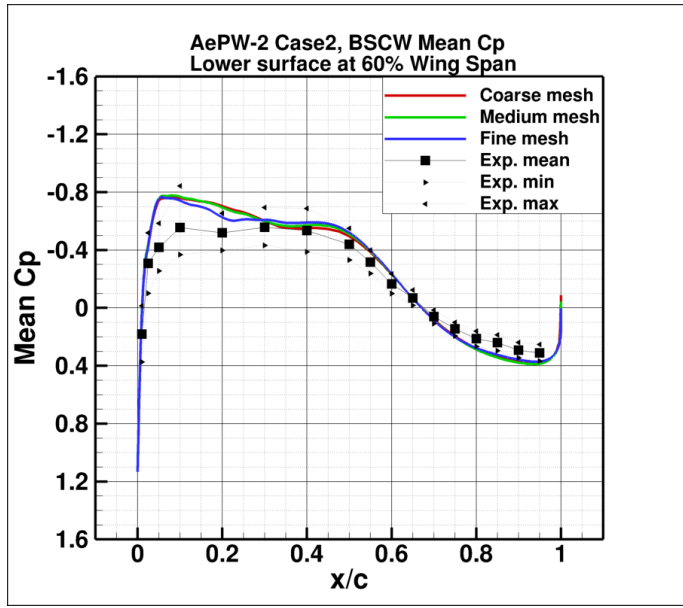

(b) Mean $C_{p}$, Lower Surface.

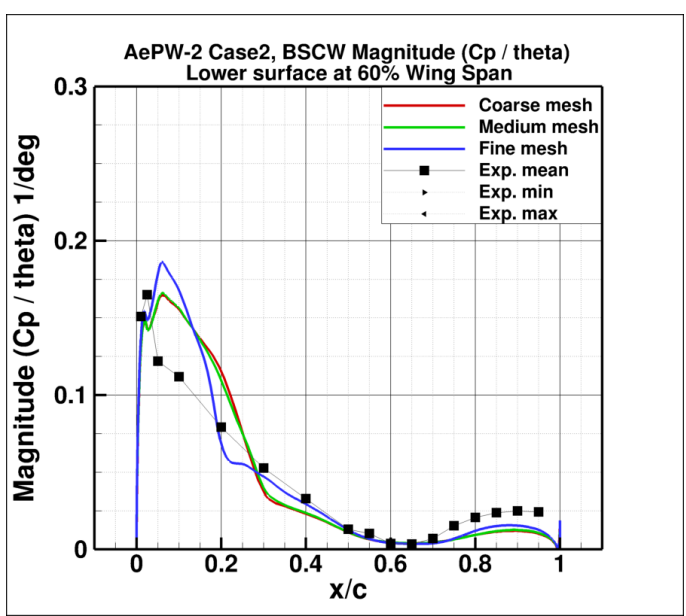

(d) $\mid C_{p} /$ theta $\mid$, Lower Surface.

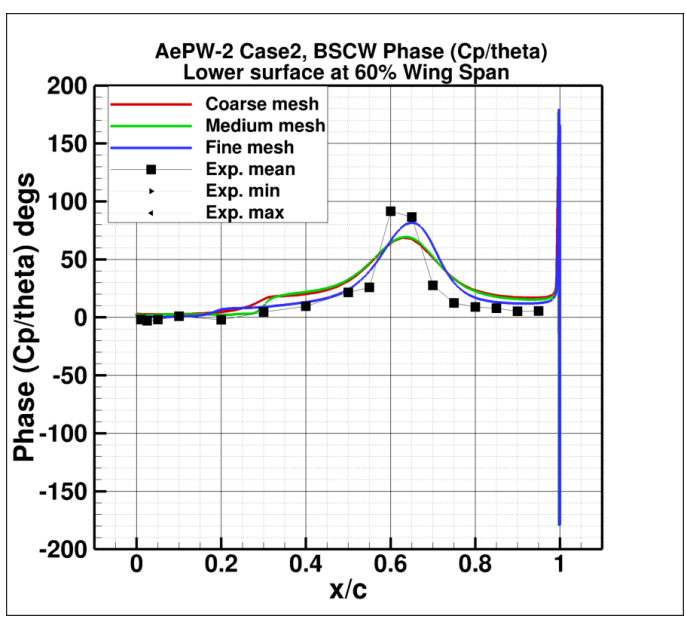

(f) Phase $C_{p}$ /theta, Lower Surface.

Figure 5. Mean $C_{p}$ and Frequency Response Function: Magnitude and Phase of Pressure Due to Pitch Angle, Mach 0.74, $\mathrm{q}=168.8 \mathrm{psf}, \operatorname{Re}_{c}=4.45 * 10^{6}, \alpha=0^{\circ}, \mathbf{6 0} \%$ Wing Span, $f^{*}=$ [4.1 Hz (coarse), 4.1 Hz (medium), 4.1 (fine)]. 


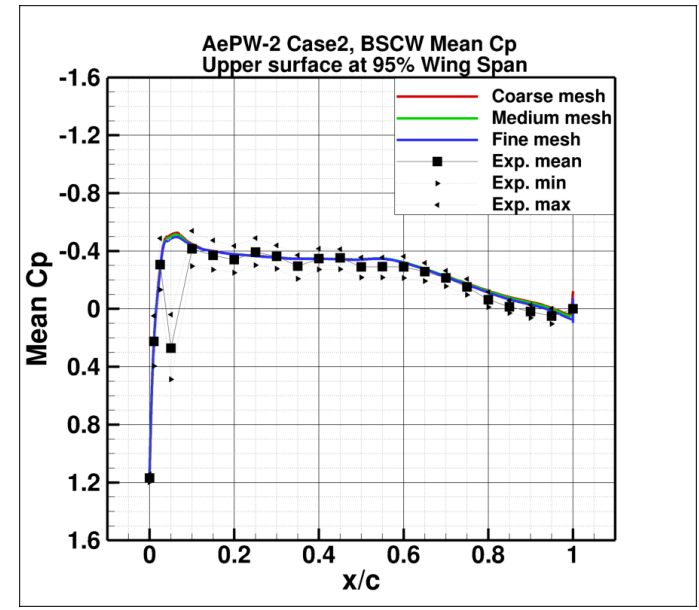

(a) Mean $C_{p}$, Upper Surface.

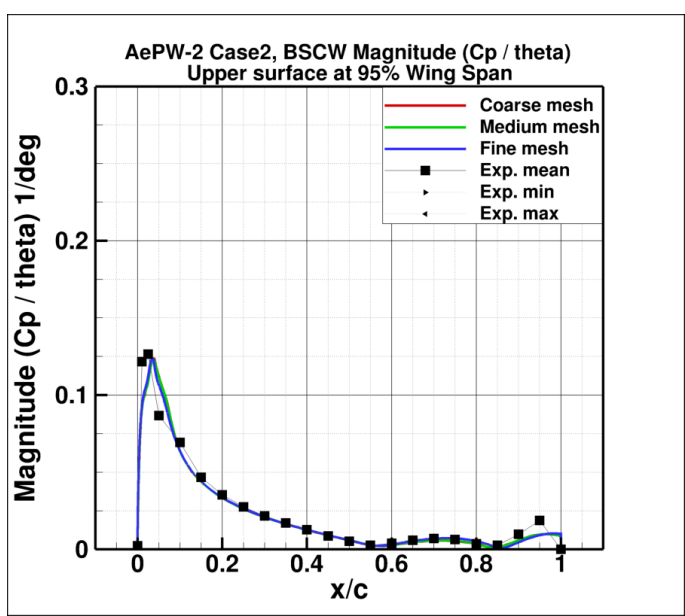

(c) $\mid C_{p} /$ theta $\mid$, Upper Surface.

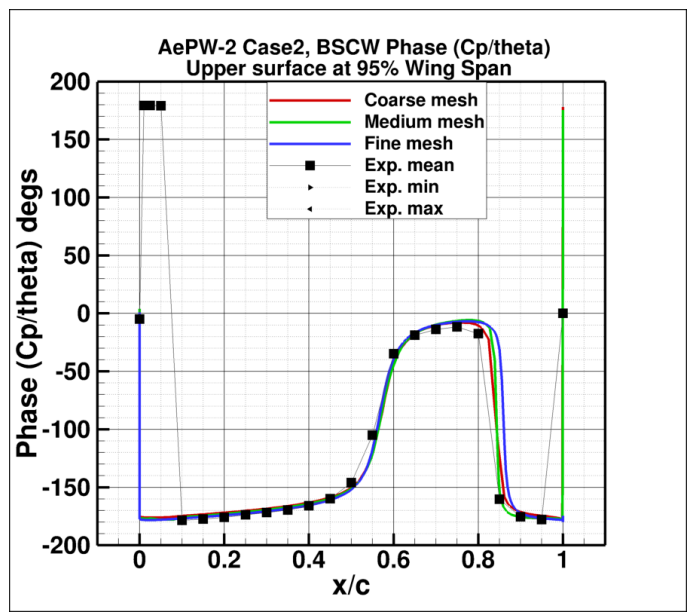

(e) Phase $C_{p} /$ theta, Upper Surface.

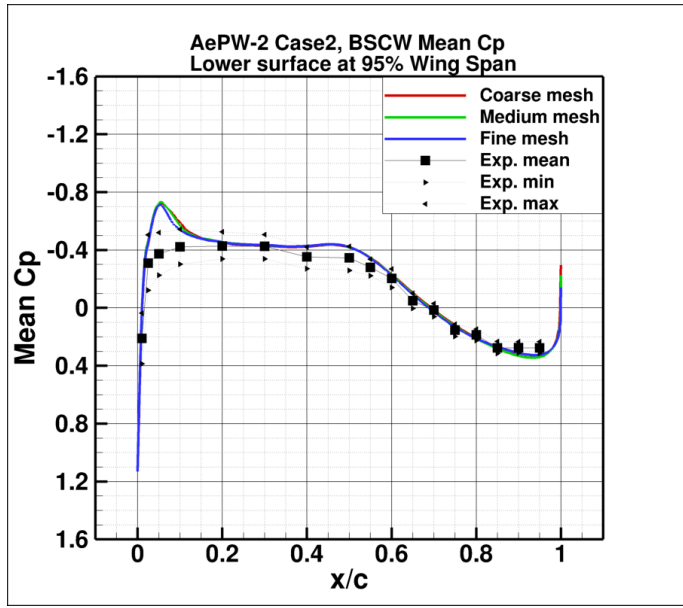

(b) Mean $C_{p}$, Lower Surface.

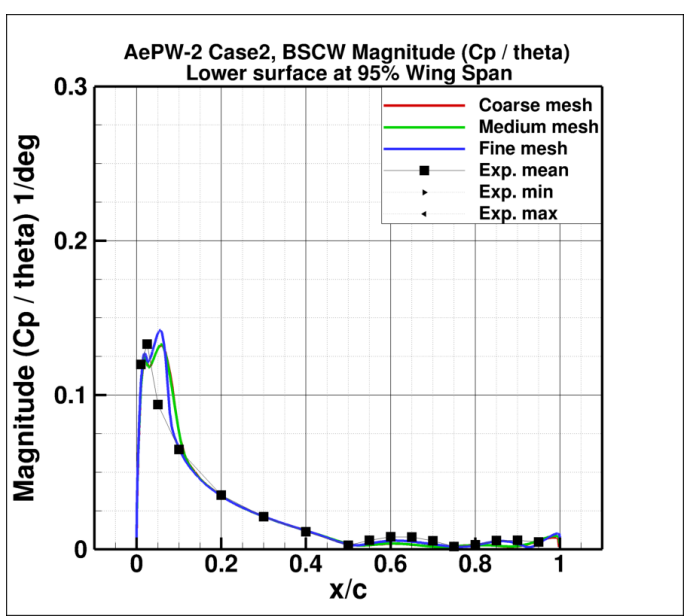

(d) $\mid C_{p} /$ theta $\mid$, Lower Surface.

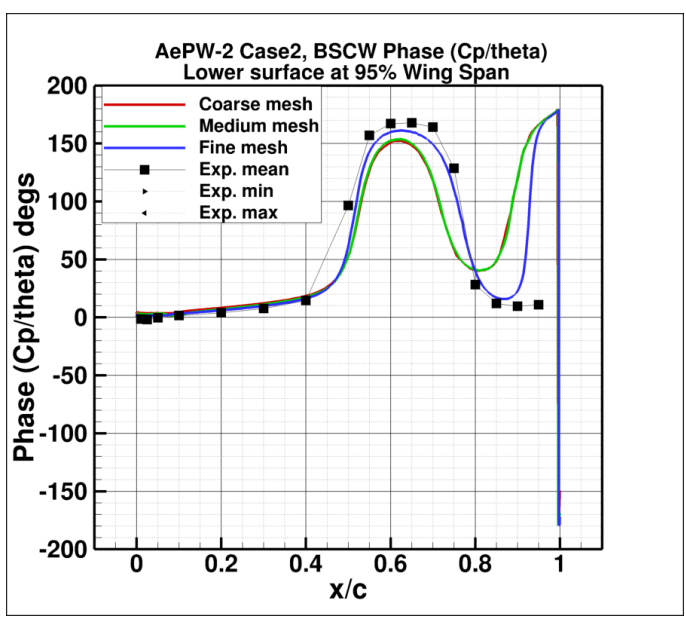

(f) Phase $C_{p}$ /theta, Lower Surface.

Figure 6. Mean $C_{p}$ and Frequency Response Function: Magnitude and Phase of Pressure Due to Pitch Angle, Mach 0.74, $\mathrm{q}=168.8 \mathrm{psf}, \operatorname{Re}_{c}=4.45 * 10^{6}, \alpha=0^{\circ}, 95 \%$ Wing Span, $f^{*}=$ [4.1 Hz (coarse), 4.1 Hz (medium), 4.1 (fine)]. 


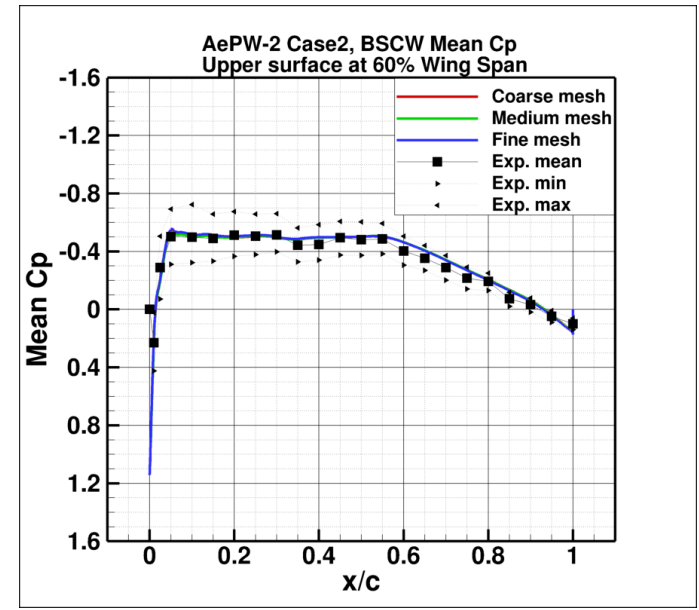

(a) Mean $C_{p}$, Upper Surface.

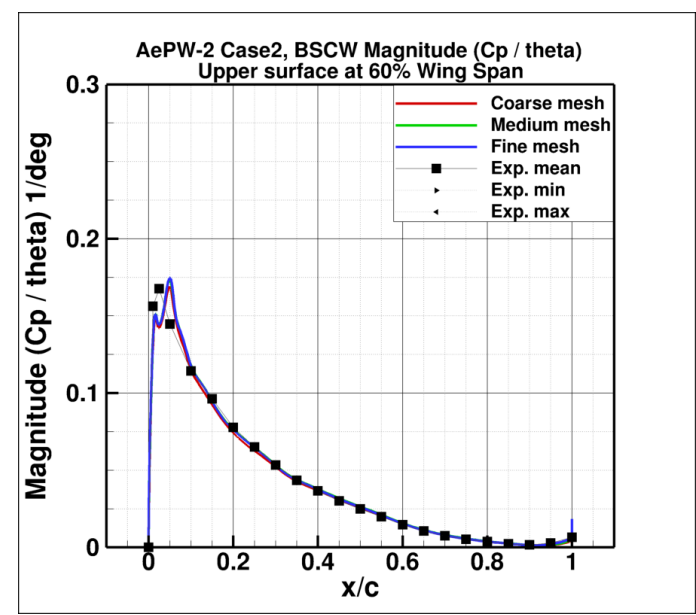

(c) $\mid C_{p} /$ theta $\mid$, Upper Surface.

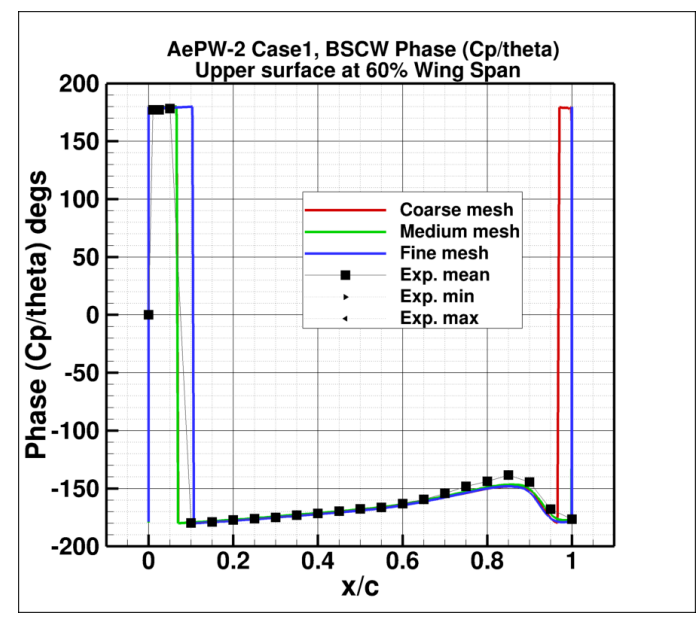

(e) Phase $C_{p} /$ theta, Upper Surface.

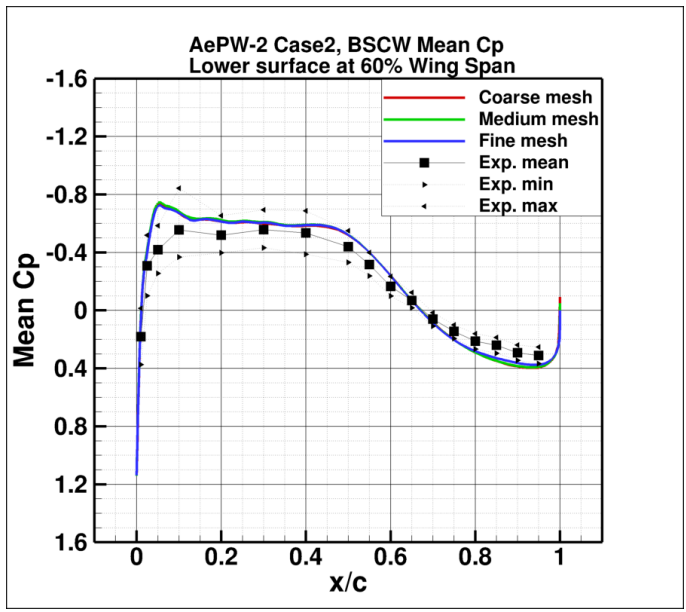

(b) Mean $C_{p}$, Lower Surface.

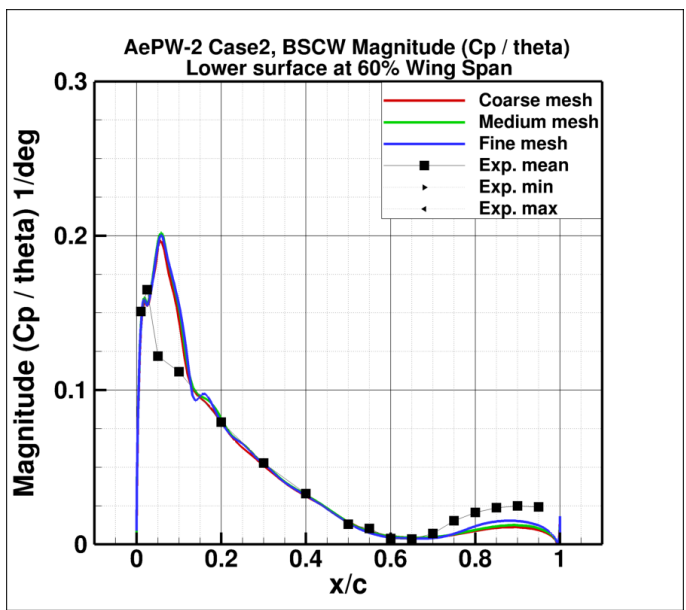

(d) $\mid C_{p} /$ theta $\mid$, Lower Surface.

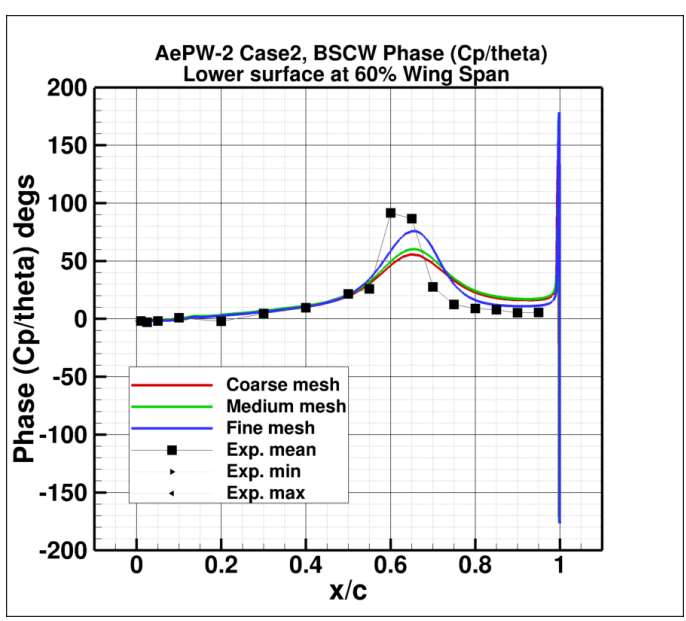

(f) Phase $C_{p} /$ theta, Lower Surface.

Figure 7. Mean $C_{p}$ and Frequency Response Function: Magnitude and Phase of Pressure Due to Pitch Angle, Mach 0.74, $q=152.0$ psf, $R e_{c}=4.45 * 10^{6}, \alpha=0^{\circ}, 60 \%$ Wing Span; Experimental Data at $q=168.8 \mathrm{psf}, f^{*}=[4.2 \mathrm{~Hz}(\operatorname{coarse}), 4.2 \mathrm{~Hz}$ (medium), 4.1 (fine)]. 


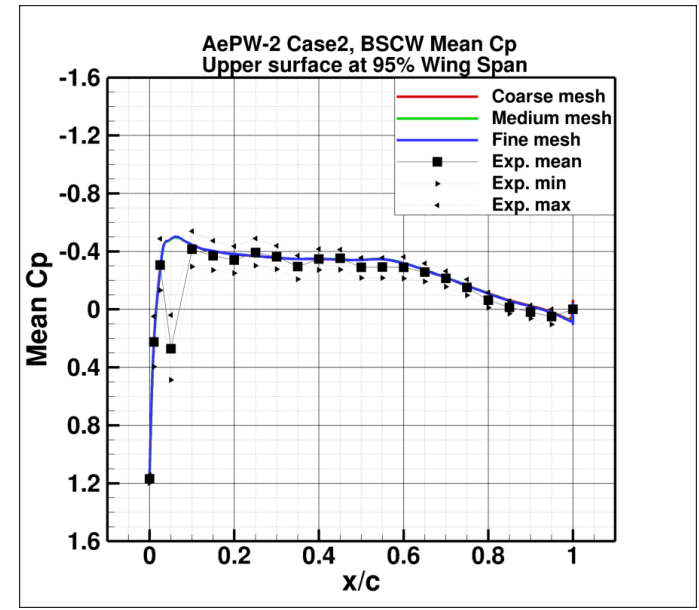

(a) Mean $C_{p}$, Upper Surface.

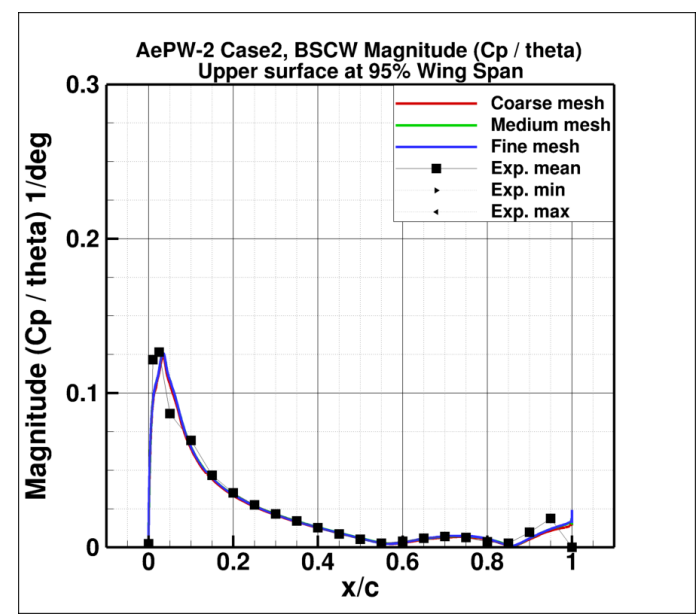

(c) $\mid C_{p} /$ theta $\mid$, Upper Surface.

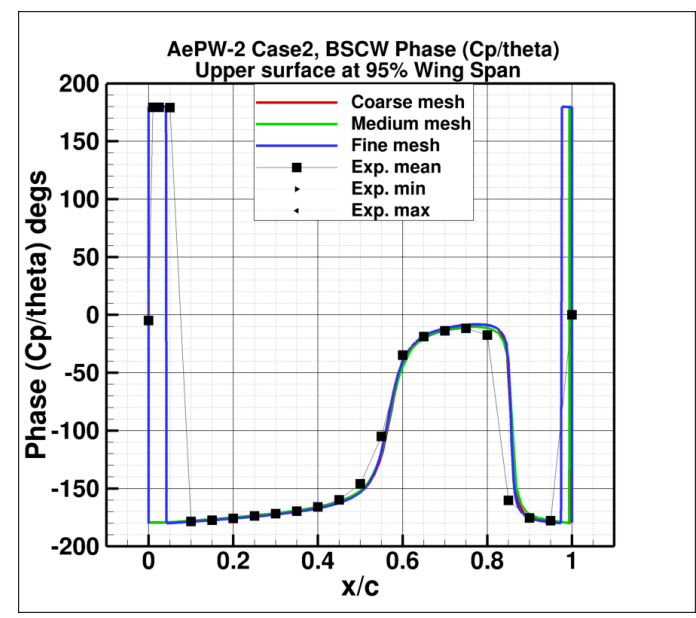

(e) Phase $C_{p}$ /theta, Upper Surface.

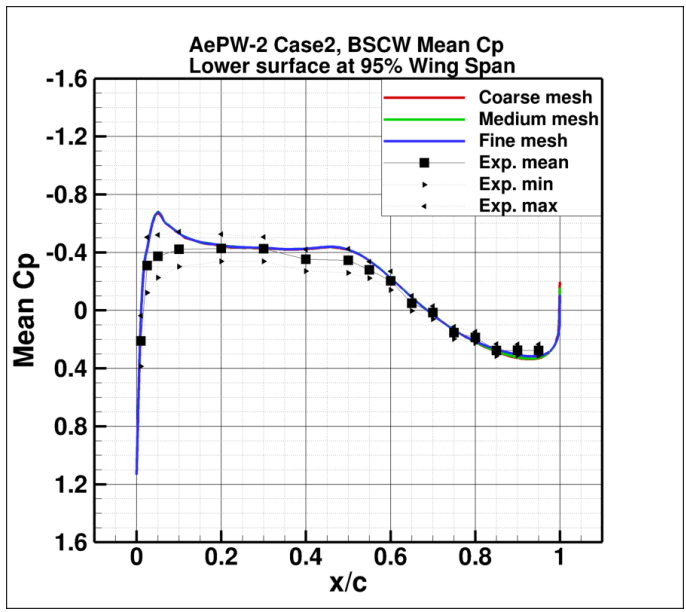

(b) Mean $C_{p}$, Lower Surface.

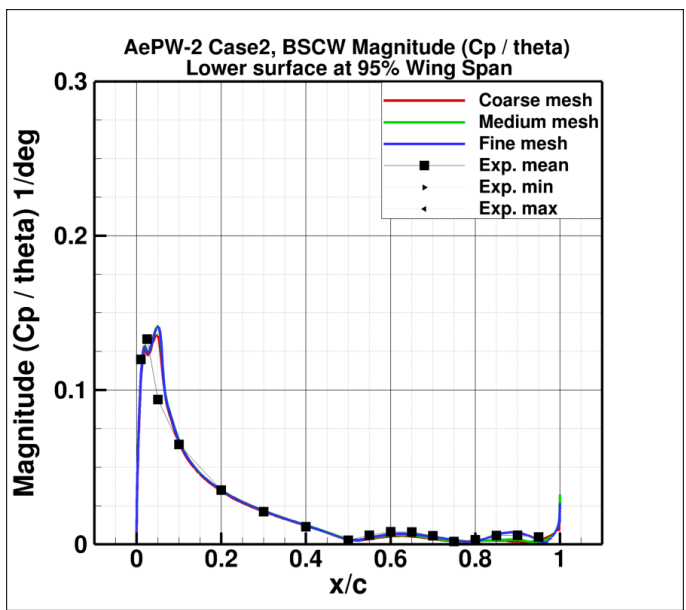

(d) $\mid C_{p} /$ theta $\mid$, Lower Surface.

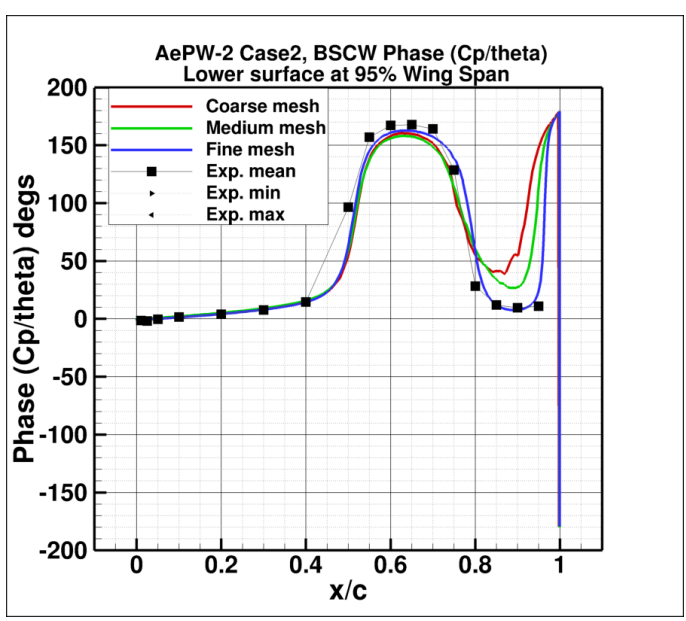

(f) Phase $C_{p} /$ theta, Lower Surface.

Figure 8. Mean $C_{p}$ and Frequency Response Function: Magnitude and Phase of Pressure Due to Pitch Angle, Mach 0.74, $q=152.0$ psf, $R e_{c}=4.45 * 10^{6}, \alpha=0^{\circ}, 95 \%$ Wing Span; Experimental Data at $q=168.8 \mathrm{psf}, f^{*}=[4.2 \mathrm{~Hz}(\operatorname{coarse}), 4.2 \mathrm{~Hz}$ (medium), 4.1 (fine)]. 
One of the most important lessons learned from analysis of the BSCW test case for AePW-1 was that more information on temporal convergence of both the unforced and the forced oscillation solutions was needed in order to assess the quality of "goodness" of the results. Subsequent analyses have shown great sensitivity to temporal convergence, including changing the system from stable (flutter-free) to unstable (flutter). Understanding temporal convergence criteria was therefore a high priority for AePW-2. The paragraph below briefly describes issues associated with the selection of the global time step size, the specification of the number of subiterations, and the convergence criteria at the subiteration level in FUN3D for Case \#2 of AePW-2.

Figure 9a shows the generalized displacements for Modes 1 and 2 when FUN3D was executed with three levels of non-dimensional time-step sizes (DT) corresponding to dimensional time-step sizes of 0.0002, 0.002, and 0.004 seconds. At these time steps, the solution behavior changes from stable ( $\mathrm{dt}=0.004$ seconds - green curve), to slightly unstable ( $\mathrm{dt}=0.002$ seconds - black curve), to unstable ( $\mathrm{dt}=0.0002$ seconds - red curve). All the solutions were obtained by running FUN3D on a coarse grid with 15 subiterations at each global time step. Figure $9 \mathrm{~b}$ shows the continuation of the temporal convergence study, where in addition to the solution with the time step of 0.0002 seconds (red curve; which is not visible because it is very close to the blue curve), two additional solutions were added. One was obtained with the same $\mathrm{dt}=0.0002$ seconds but with 1000 subiterations (blue curve). The second solution was obtained by reducing the time-step size to 0.00002 seconds but the number of subiterations was kept at 15 (orange curve). These three solutions produced nearly identical results. Therefore, it is concluded that the FUN3D solution on a coarse grid obtained with a time-step size of 0.0002 seconds and 15 subiterations (or more) is sufficient for Case \#2 flutter prediction. Similar analyses were performed running FUN3D on medium and fine grids. Those results showed that a minimum of 25 subiterations with the time step of 0.0002 seconds are needed for Case \#2 flutter prediction. Further investigation of these solutions showed that different convergence levels at the subiteration level were obtained during wing-motion cycle. Typically, when the actual angle of attack was at the highest value during the cycle, more subiterations were needed to drop the residuals to the satisfactory level than when the angle of attack was small. This observation led to additional analysis described below.

The FUN3D solver optionally employs a temporal error control parameter to provide a residual-cutoff region for the solution to stop at the subiteration level and to proceed to the next global time step. This cutoff occurs when the residuals drop below the product of that parameter and an estimate of the temporal error. In a typical BSCW FUN3D computation, we specify 1000 subiterations and a temporal error control parameter of 0.1 . This in turn resulted in about 50 to 150 subiterations cutoff at each global time step. Further studies will evaluate the effect of the magnitude of the temporal error control parameter on the flutter prediction.

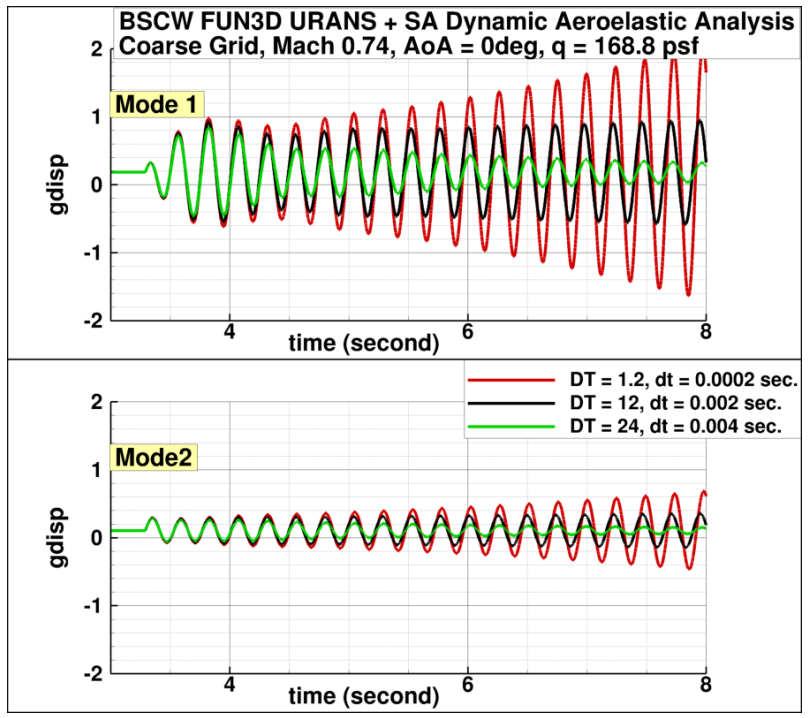

(a) Computed Generalized Displacements at Three Time-Step Levels $(\mathrm{DT}=1.2,12,24)$ and 15 subiterations.

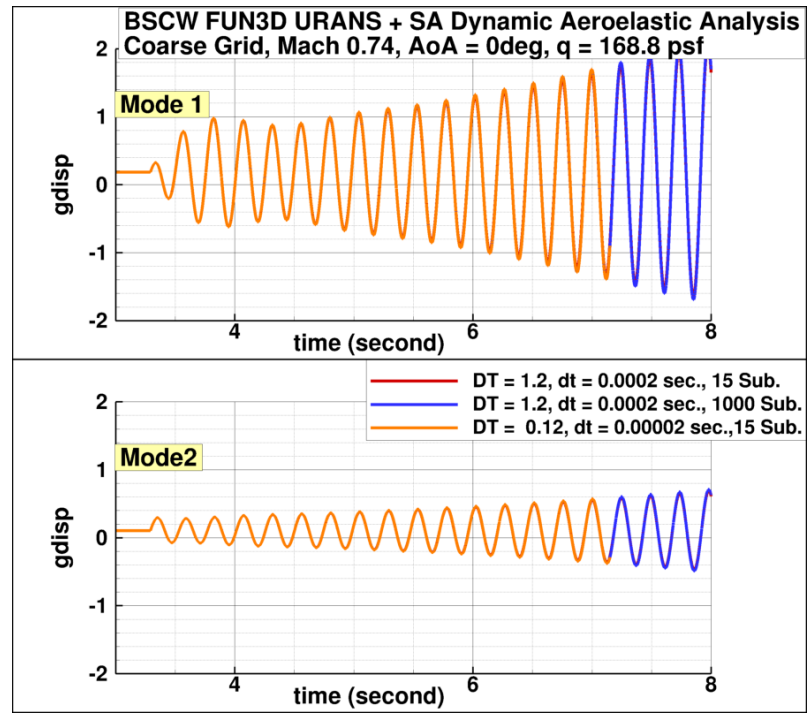

(b) Computed Generalized Displacements at Two Time-Step Levels (DT $=1.2,0.12$ ) and 15 and 1000 subiterations.

Figure 9. Time History of the Generalized Displacements at Various Time-Step Sizes, BSCW, Mach $0.74, q=168.8$ psf, $R e_{c}$ $=4.45 * 10^{6}, \alpha=0^{\circ}$. 


\section{Test Case \#3}

Test Case \#3 is an optional workshop case that is a carry over from AePW-1. This case focused on the BSCW model at Mach $0.85,5^{\circ}$ angle of attack. The AePW-1 results showed significant scatter in the data reported by the different analysis teams. Based on the AePW-1 results and recommendations from the community, this case was split into three parts. The first sub-case, designated Case \#3a here, assessed the rigid-steady versus rigid-unsteady flow calculations in the presence of the shock-induced separated flow, which dominates the upper surface and the aft portion of the lower surface. The second sub-case, designated Case \#3b, computed the flow around the wing undergoing forced oscillation at $10 \mathrm{~Hz}$. For both Cases, \#3a and \#3b, some experimental data is available. The most challenging and difficult sub-case, designated Case \#3c, was the flutter onset prediction at this condition. Unfortunately, experimental data does not exist for this case; so, it is considered to be a 'blind' test case.

\section{Test Case \#3a}

This case assessed the unforced (or rigid) steady-state solutions and the unforced time-accurate solutions. The results discussed and presented here are for the medium-grid resolution. Tables 7, 8, and 9 list the computed mean aerodynamic coefficients with relevant statistical information. The statistical information was computed from the solution by excluding the initial solution transient. For the data shown in Tables 7 and 9, FUN3D was run without a limiter, while one was used for computing the data in Table 8 . The difference in mean aerodynamic coefficients values between the solutions is significant. Figure 10a shows the lift and pitching moment coefficients obtained during solution development. For the unforced steady-state solutions, the true steady state is never reached. This is because of the shock movement and its interaction with the separated flow behind the shock. The unforced time-accurate solution follows the unforced steady solution.

The mean shock location on the upper surface at $60 \%$ wing span is computed at different locations for solutions with and without a limiter as presented in Figure 10b. It will be shown in the Case \#3c analysis section of this paper that the shock location has a significant influence on the flutter prediction. Figure10b also shows small differences in $C_{p}$ values on the upper and lower surfaces aft of the shock at $60 \%$ and $95 \%$ span stations for solutions with and without a limiter. In general, the limiter adds additional numerical damping to stabilize the solution near strong gradients like shocks. We believe that it is appropriate not to introduce this additional damping; nevertheless, we are showing both solutions.

Table 7. Test Case \#3a: BSCW Analysis Aerodynamic Coefficients at Mach 0.85, $\alpha=5^{\circ}$, Medium Grid, FUN3D Unforced Steady-State Solution without Limiter.

\begin{tabular}{c|c|c|c|c|c|c|c|c|c}
\hline \hline & $C_{L}$ & $C_{D}$ & $C_{M y}$ & $C_{l 60}$ & $C_{d 60}$ & $C_{m y 60}$ & $C_{l 95}$ & $C_{d 95}$ & $C_{m y 95}$ \\
\hline Mean & 0.3946 & 0.0755 & -0.0276 & 0.4408 & 0.0783 & -0.0326 & 0.2991 & 0.0541 & -0.0331 \\
\hline Mode & 0.3858 & 0.0749 & -0.0293 & 0.4036 & 0.0752 & -0.0406 & 0.2906 & 0.0532 & -0.0343 \\
\hline Median & 0.3945 & 0.0755 & -0.0278 & 0.4495 & 0.0785 & -0.0341 & 0.300 & 0.0543 & -0.0335 \\
\hline Min & 0.3858 & 0.0749 & -0.0293 & 0.4036 & 0.0752 & -0.0406 & 0.2906 & 0.0532 & -0.0343 \\
\hline Max & 0.4016 & 0.0760 & -0.0261 & 0.4659 & 0.0808 & -0.0229 & 0.3034 & 0.0546 & -0.0311 \\
\hline Std & 0.0042 & 0.0002 & 0.0009 & 0.0209 & 0.0015 & 0.0053 & 0.0040 & 0.0004 & 0.0009 \\
\hline \hline
\end{tabular}

Table 8. Test Case \#3a: BSCW Analysis Aerodynamic Coefficients at Mach 0.85, $\alpha=5^{\circ}$, Medium Grid, FUN3D Unforced Steady-State Solution with Limiter.

\begin{tabular}{c|c|c|c|c|c|c|c|c|c}
\hline \hline & $C_{L}$ & $C_{D}$ & $C_{M y}$ & $C_{l 60}$ & $C_{d 60}$ & $C_{m y 60}$ & $C_{l 95}$ & $C_{d 95}$ & $C_{m y 95}$ \\
\hline Mean & 0.4245 & 0.0776 & -0.0367 & 0.4775 & 0.0800 & -0.0421 & 0.3128 & 0.0568 & -0.0378 \\
\hline Mode & 0.4320 & 0.0778 & -0.0388 & 0.4955 & 0.0792 & -0.0411 & 0.3182 & 0.0576 & -0.0396 \\
\hline Median & 0.4263 & 0.0777 & -0.0372 & 0.4801 & 0.0801 & -0.0415 & 0.3137 & 0.0569 & -0.0380 \\
\hline Min & 0.4046 & 0.0768 & -0.0388 & 0.4380 & 0.0791 & -0.0453 & 0.3001 & 0.0552 & -0.0396 \\
\hline Max & 0.4333 & 0.0780 & -0.0315 & 0.4955 & 0.0810 & -0.0389 & 0.3187 & 0.0576 & -0.0341 \\
\hline Std & 0.0083 & 0.0003 & 0.0022 & 0.0163 & 0.0004 & 0.0020 & 0.0053 & 0.0006 & 0.0015 \\
\hline \hline
\end{tabular}


Table 9. Test Case \#3a: BSCW Analysis Aerodynamic Coefficients at Mach 0.85, $\alpha=5^{\circ}$, Medium Grid, FUN3D Unforced Time-Accurate Solution without Limiter.

\begin{tabular}{c|c|c|c|c|c|c|c|c|c}
\hline \hline & $C_{L}$ & $C_{D}$ & $C_{M y}$ & $C_{l 60}$ & $C_{d 60}$ & $C_{m y 60}$ & $C_{l 95}$ & $C_{d 95}$ & $C_{m y 95}$ \\
\hline Mean & 0.3942 & 0.0756 & -0.0277 & 0.4376 & 0.0783 & -0.0315 & 0.2983 & 0.0540 & -0.0329 \\
\hline Mode & 0.3821 & 0.0750 & -0.0295 & 0.4123 & 0.0747 & -0.0378 & 0.3021 & 0.0534 & -0.0341 \\
\hline Median & 0.3949 & 0.0756 & -0.0278 & 0.4386 & 0.0783 & -0.0319 & 0.2990 & 0.0541 & -0.0330 \\
\hline Min & 0.3821 & 0.0750 & -0.0295 & 0.3992 & 0.0741 & -0.0435 & 0.2908 & 0.0533 & -0.0346 \\
\hline Max & 0.4031 & 0.0761 & -0.0251 & 0.4684 & 0.0821 & -0.0185 & 0.3046 & 0.0547 & -0.0309 \\
\hline Std & 0.0053 & 0.0002 & 0.0010 & 0.0149 & 0.0016 & 0.0056 & 0.0039 & 0.0004 & 0.0010 \\
\hline \hline
\end{tabular}

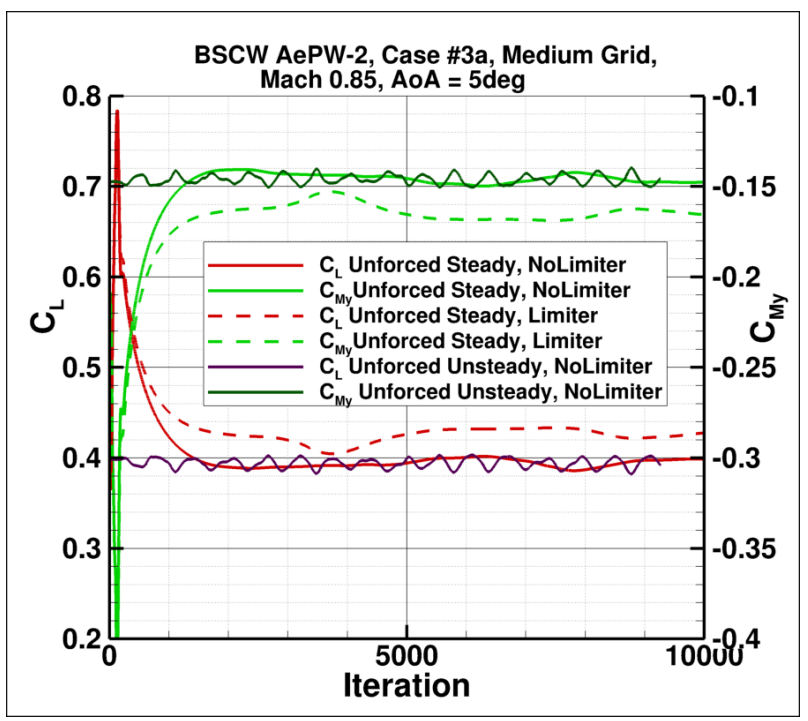

(a) Lift and Pitching Moment Coefficients, FUN3D Solutions with and without Limiter.

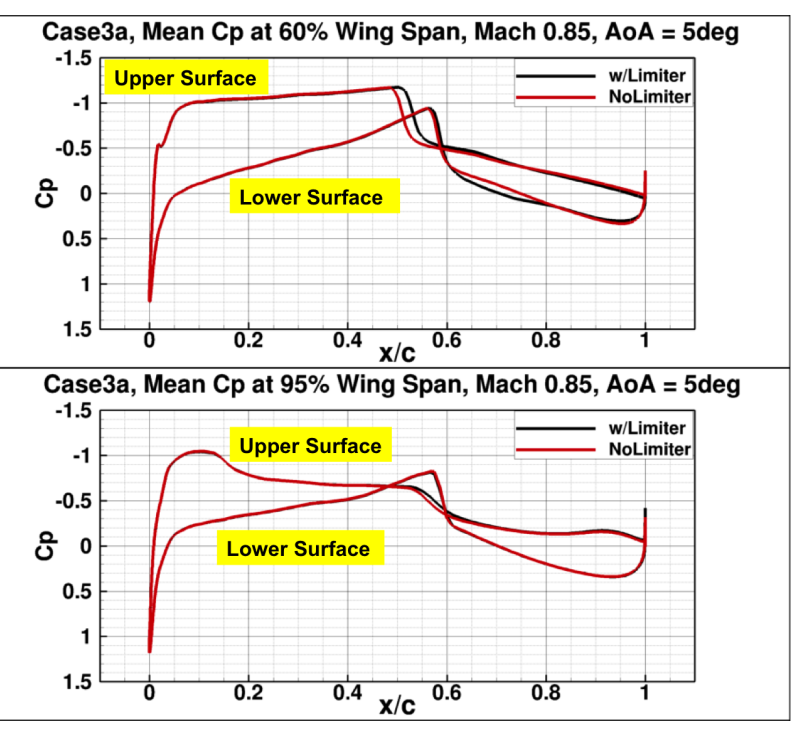

(b) Mean $C_{p}$, at $60 \%$ and $95 \%$ Wing Span.

Figure 10. Aerodynamic Coefficients and Mean $C_{p}$ at $60 \%$ and 95\% Wing Span, Case \#3a, Mach $0.85, R e_{c}=4.49 * 10^{6}$, $\alpha=0^{\circ}$.

\section{Test Case \#3b}

This case involved forced-pitch oscillation at $10 \mathrm{~Hz}$ with a pitch amplitude of 1 degree. The same time-step size, $\mathrm{dt}=$ 0.0002, was used for all grids with the same temporal convergence error set to 0.1. Figures 11(a)-(f) show the BSCW computational results generated at the $60 \%$ wing-span station. Figures 11 (a)-(b) provide the mean $C_{p}$ values obtained for the upper and lower surfaces, respectively. The numerically-computed shock location on the upper surface is aft of the experimental shock for the FUN3D solution with SA turbulence model and forward of it for the DDES method. There is poor agreement in pressure aft of the shock. This can be attributed to the inability of the RANS solver to correctly capture flow physics in the separated-flow region. For the lower surface, Figure 11(b), it is assumed that the resolution of pressure taps was too sparse to experimentally capture the shock location. Figures 11(c) and (d) show the magnitude of the FRF of pressure due to angular displacement, and Figures 11(e) and (f) show the corresponding phase of the FRF. Interestingly, the magnitude of the FRF (DDES solution) captures the shock location quite well. This suggests that perhaps the mean $C_{p}$ is not a good quantity representation of the average shock location.

\section{Test Case \#3c}

This third optional test case required computation of the flutter boundary at Mach 0.85 and $5^{\circ}$ angle of attack. The process for finding the flutter boundary is the same as that outlined and used for Case \#2. Figure 12 graphically demonstrates the issues and various solutions associated with computation of the flutter boundary for this case. First of all, depending on the grid resolution and the scheme used, the dynamic pressure range is nearly 280 psf for the 


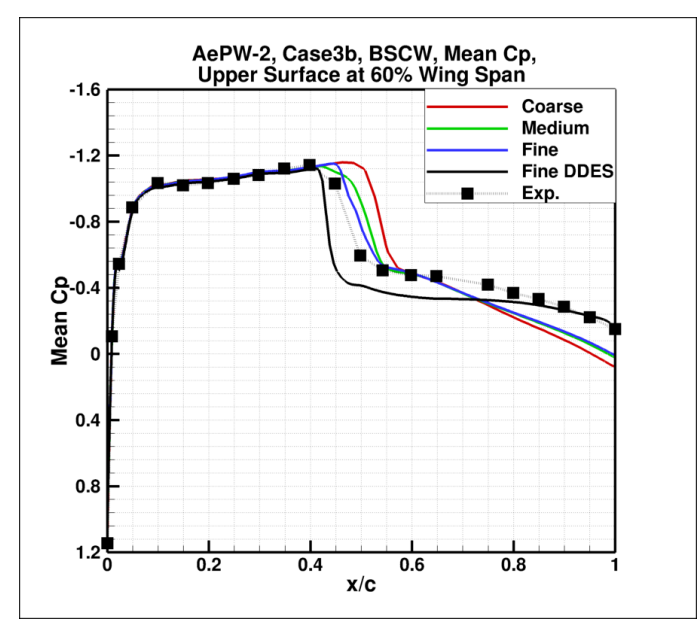

(a) Mean $C_{p}$, Upper Surface.

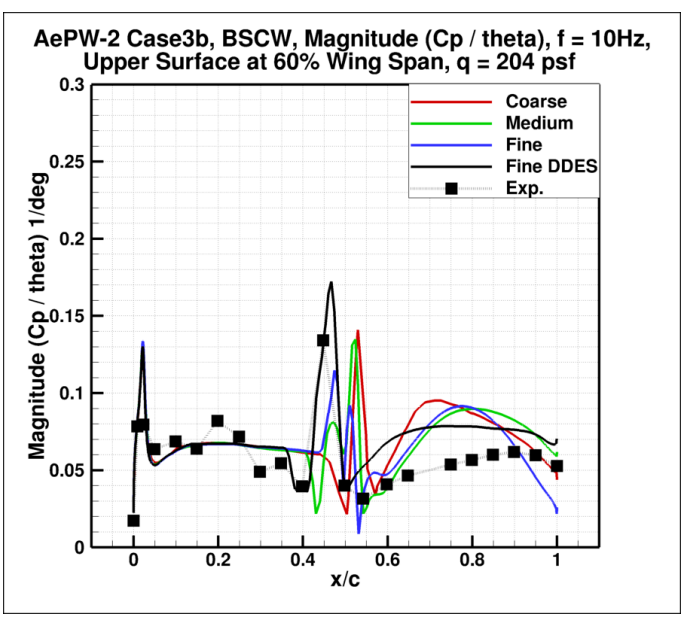

(c) $\mid C_{p} /$ theta $\mid$, Upper Surface.

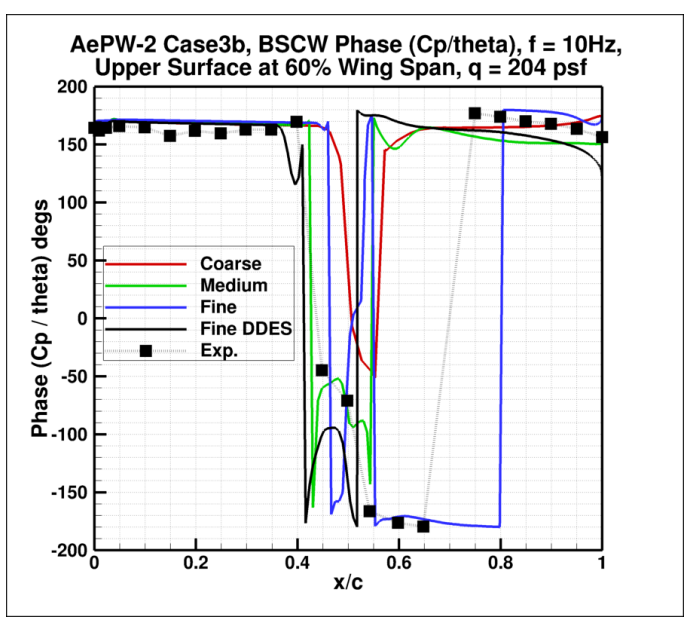

(e) Phase $C_{p} /$ theta, Upper Surface.

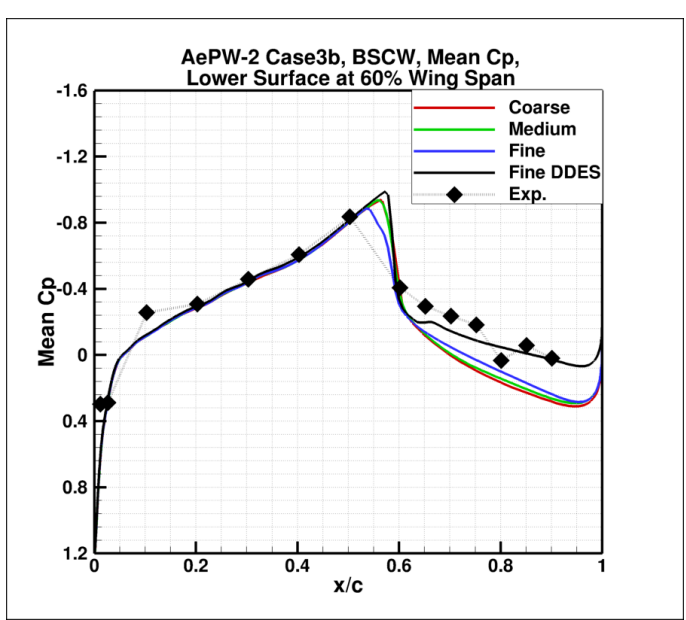

(b) Mean $C_{p}$, Lower Surface.

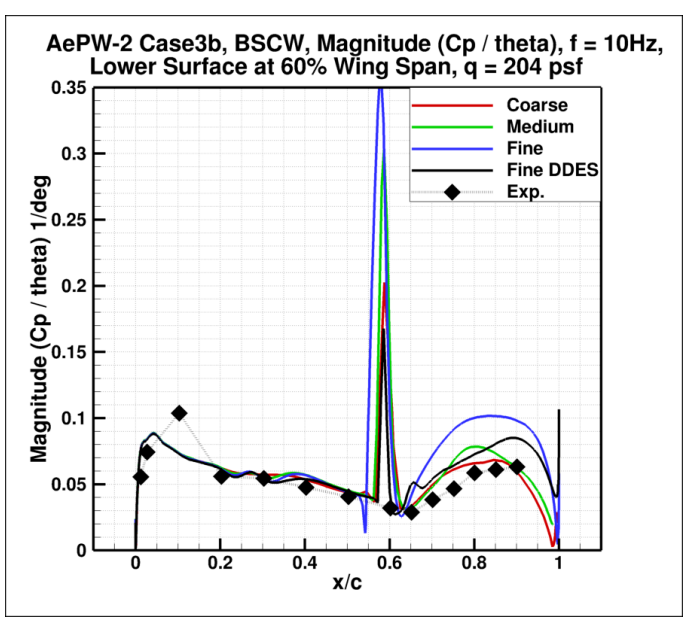

(d) $\mid C_{p} /$ theta $\mid$, Lower Surface.

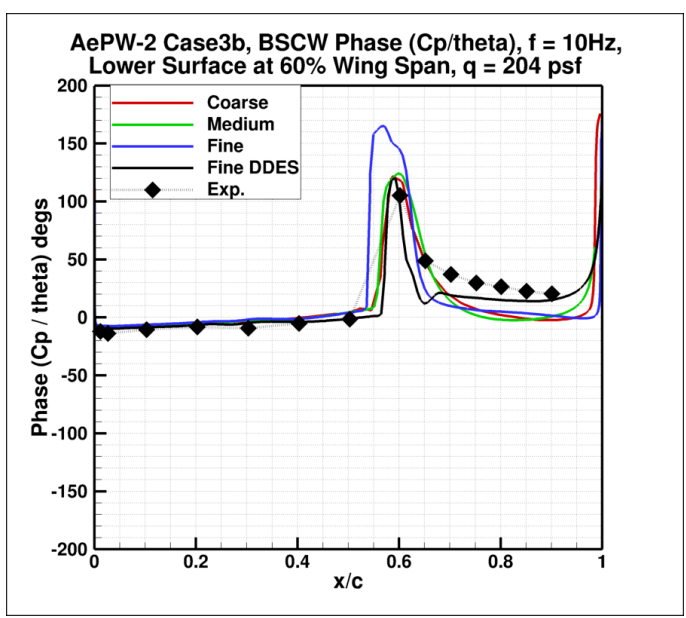

(f) Phase $C_{p}$ /theta, Lower Surface.

Figure 11. Mean $C_{p}$ and Frequency Response Function: Magnitude and Phase of Pressure Due to Pitch Angle, Mach 0.85, $\mathrm{q}=204 \mathrm{psf}, \mathrm{f}=10 \mathrm{~Hz}, \operatorname{Re}_{c}=4.49 * 10^{6}, \alpha=5^{\circ}, 60 \%$ Wing Span. 
flutter boundary prediction at Mach 0.85 . This is a very wide range and clearly requires further examination.

As mentioned previously in the discussion of Case \#3a, the application of a limiter in the solution process shifts the predicted shock location aft on the upper surface of the wing, affecting the size of the separated region behind the shock. The grid resolution affects shock strength and spatial distribution. As shown in Figure 12, the range of the dynamic pressure between the coarse and fine grid solutions with a limiter is nearly $200 \mathrm{psf}$. On the other hand, the corresponding range for the solutions without the limiter is only about $100 \mathrm{psf}$. In addition, a DDES solution on a fine grid produced a flutter boundary point in between the coarse- and fine-grid solutions. This very wide range of predictions prompted an attempt to calculate the flutter boundary over a wide range of Mach numbers from 0.6 to 0.85 at $5^{\circ}$ angle of attack.

The calculation at Mach 0.82 on a coarse grid produced a flutter boundary point at $200 \mathrm{psf}$. This calculation will be verified on a fine grid. The coarse grid calculations at Mach number of 0.8 and 0.74 did not establish flutter boundary predictions at all. All solutions were always unstable, even with the dynamic pressure of $25 \mathrm{psf}$. On the other hand, the calculations at Mach number of 0.6 and 0.7 predicted nearly identical flutter boundary dynamic pressure of about 100 psf. The calculations at Mach 0.74 and Mach 0.8 were repeated using Grid D with the DDES turned on and off. Note that the Grid D was constructed to better resolve the wake region and to make the grid more isotropic in the separated flow region. One more grid refinement step is planned for future analysis. The calculation with the DDES turned on and off produced the same flutter boundary prediction. The solutions were stable at a dynamic pressure of $50 \mathrm{psf}$ and unstable at $100 \mathrm{psf}$, with the flutter dynamic pressures at Mach 0.8 and Mach 0.74 computed to be $55 \mathrm{psf}$ and $68 \mathrm{psf}$, respectively.

Figures 13(a)-(f) show the computational results generated at the $60 \%$ wing span station. Figures 13 (a) and (b) show the mean pressures, Figures 13(c) and (d) show the magnitude of the FRF of pressure due to angular displacement, and Figures 13(e) and (f) show the corresponding phase of FRF. As discussed previously, the numericallycomputed shock locations on the upper surface for the solutions with and without a limiter are different. The solutions with the limiter for all three grid resolutions compute shock further aft on the upper surface than the solutions without the limiter. The DDES solution and the fine grid solution without the limiter predicted the shock locations closest to the leading edge.

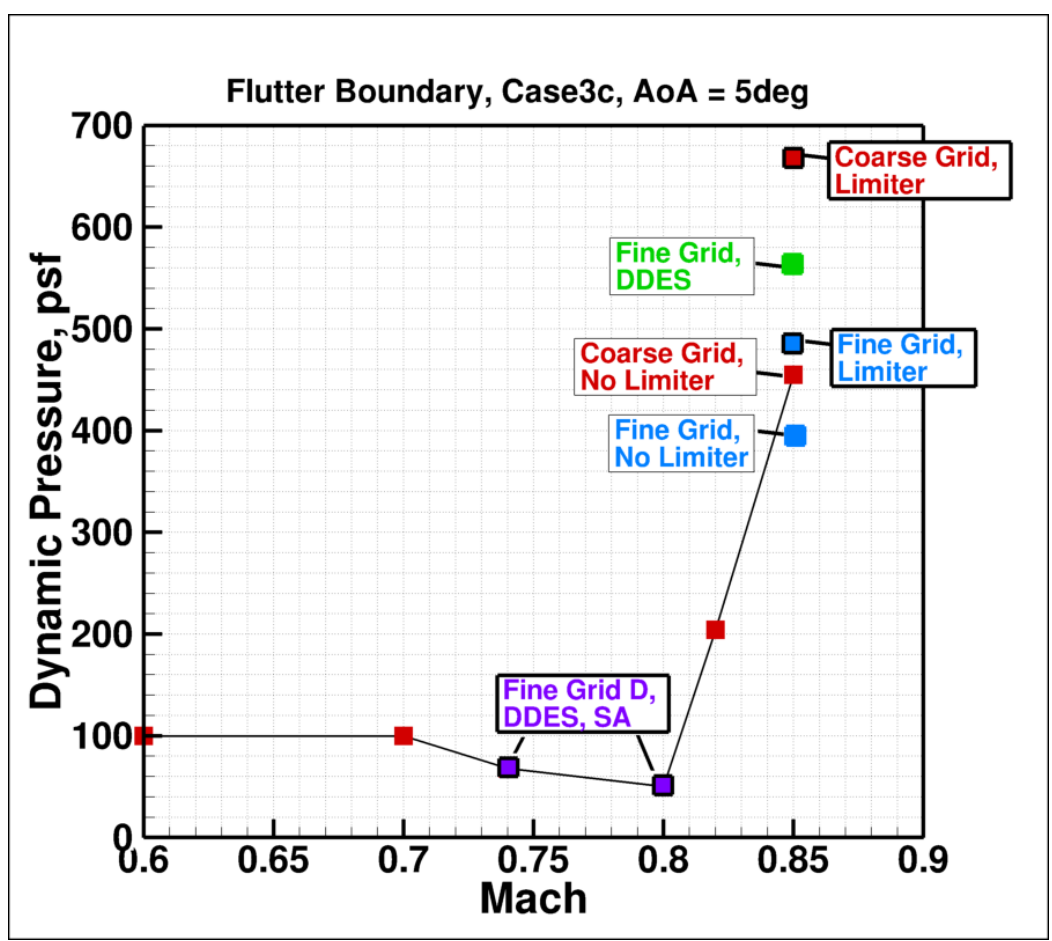

Figure 12. Flutter Boundary Assessment, BSCW Case \#3c, Mach 0.6 - 0.85, $\alpha=5^{\circ}$. 


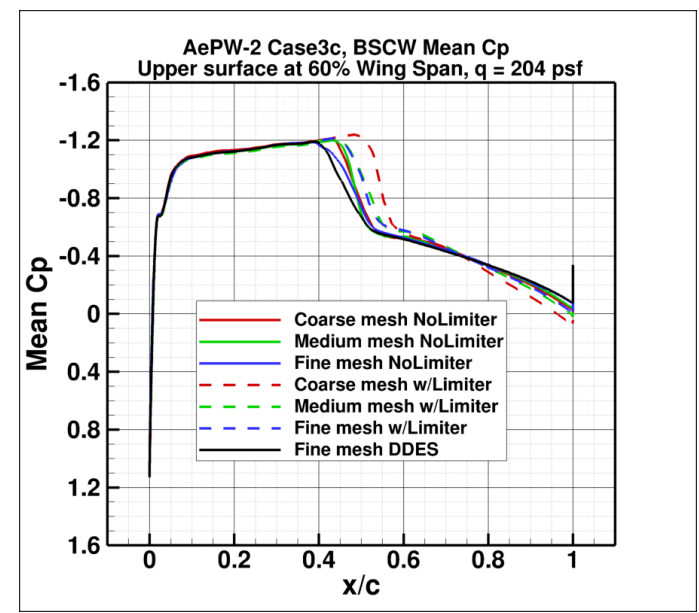

(a) Mean $C_{p}$, Upper Surface.

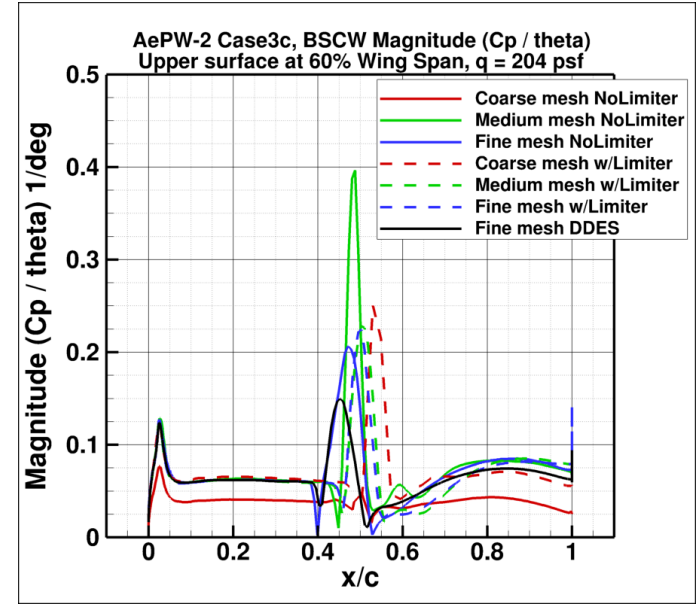

(c) $\mid C_{p} /$ theta $\mid$, Upper Surface.

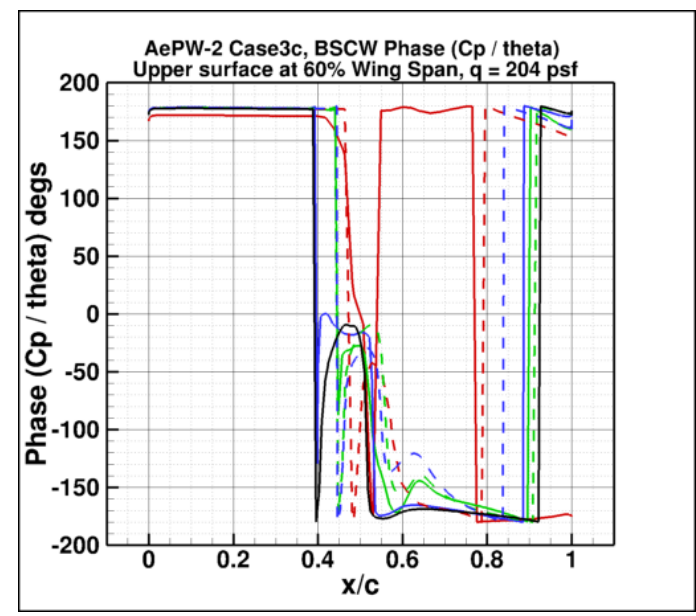

(e) Phase $C_{p}$ /theta, Upper Surface.

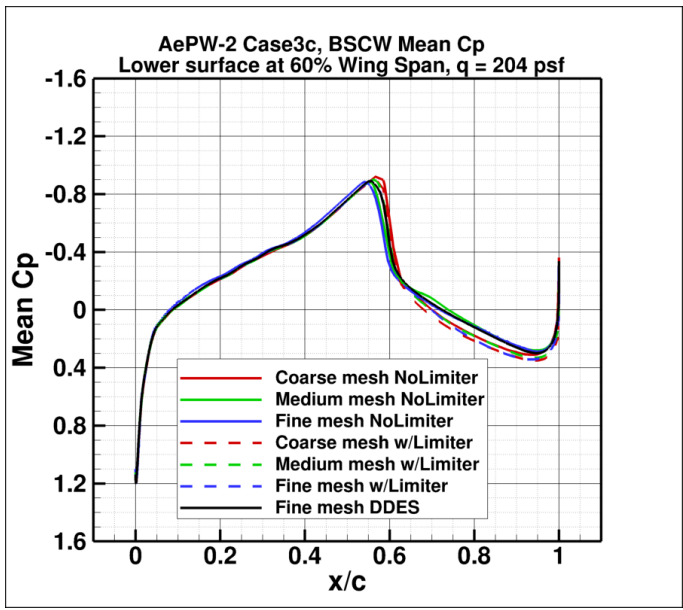

(b) Mean $C_{p}$, Lower Surface.

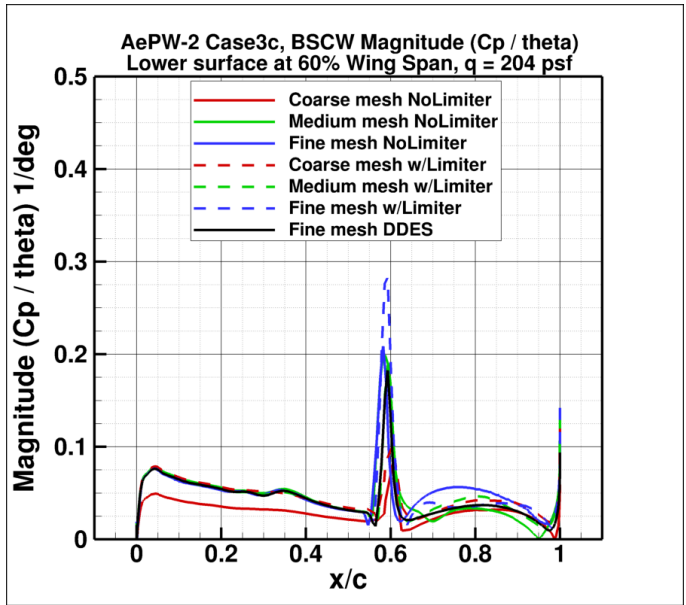

(d) $\mid C_{p}$ /theta $\mid$, Lower Surface.

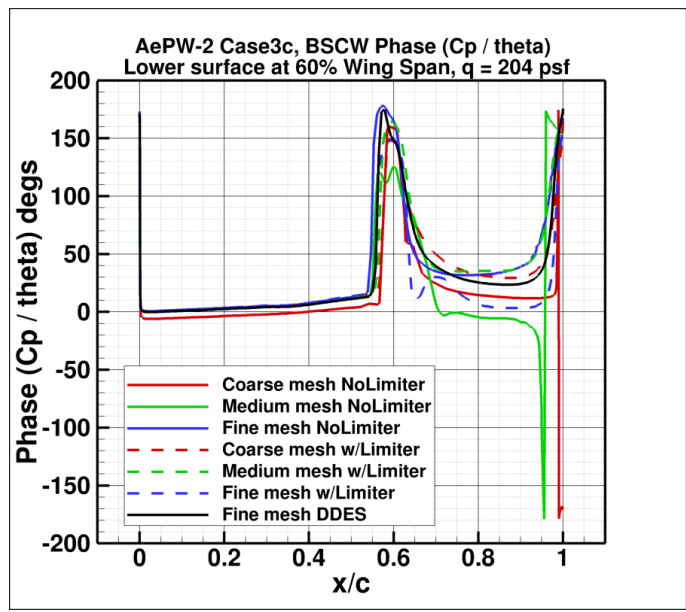

(f) Phase $C_{p}$ /theta, Lower Surface.

Figure 13. Mean $C_{p}$ and Frequency Response Function: Magnitude and phase of pressure due to pitch angle, BSCW, Mach 0.85, dynamic pressure $q=204 \mathrm{psf}, \operatorname{Re}_{c}=4.49 * 10^{6}, \alpha=5^{\circ}$, at $60 \%$ wing span, $f^{*}=[4.8 \mathrm{~Hz}$ (coarse/NoLimiter), $5.1 \mathrm{~Hz}$ (medium/NoLimiter), 5.0 Hz (fine/NoLimiter)]; [4.6 Hz (coarse/Limiter), 6.0 Hz (medium/Limiter), 4.8 Hz (fine/Limiter)]; [4.9 Hz (Fine/DDES)]. 


\section{Concluding Remarks}

Reynolds-Averaged Navier-Stokes analyses, including steady-state, static aeroelastic, forced excitation, and flutter were performed as part of AePW-2 using the NASA Langley FUN3D software. The analyses were performed on all configurations and cases considered for the workshop involving the BSCW configuration. Aerodynamic coefficients from the steady-state and time-accurate analyses were reported. When applicable, aerodynamic coefficients from the static-aeroelastic analyses were provided as well. The frequency response function of pressure due to angular displacement was calculated for the forced pitching and flutter cases and compared with the experimental data when available.

The computationally obtained flutter dynamic pressure was $10 \%$ below the experimental flutter point for Case \#2. The wide range of flutter-point prediction is reported for Case \#3c. The wide predicted range is because it is very difficult to computationally predict shock-induced separated flow for supercritical airfoils. In general, for the BSCW configuration, the computed shock location was located aft of the experimentally-measured shock location with the exception of the DDES solution. This was indicated by both the mean pressure distributions and the frequency response functions.

\section{Acknowledgments}

The authors would like to acknowledge Dr. Robert Biedron of NASA Langley for his help in setting up FUN3D post processing routines and for valuable discussions and help in understanding of application of limiters and DDES in FUN3D .

\section{References}

\footnotetext{
${ }^{1}$ Schuster, D. M., Chwalowski, P., Heeg, J., and Wieseman, C., "Summary of Data and Findings from the First Aeroelastic Prediction Workshop," International Conference on Computational Fluid Dynamics, ICCFD7 paper 3101, July 2012.

${ }^{2}$ Heeg, J., Chwalowski, P., Schuster, D. M., Dalenbring, M., Jirasek, A., Taylor, P., Mavriplis, D. J., Boucke, A., Ballmann, J., and Smith, M., "Overview and Lessons Learned from the Aeroelastic Prediction Workshop," IFASD Paper 2013-1A, June 2009.

${ }^{3}$ Heeg, J., Chwalowski, P., Schuster, D. M., and Dalenbring, M., "Overview and lessons learned from the Aeroelastic Prediction Workshop," AIAA Paper 2013-1798, April 2013.

${ }^{4}$ Heeg, J., Chwalowski, P., Schuster, D. M., Raveh, D., Jirasek, A., and Dalenbring, M., "Plans and Example Results for the 2nd AIAA Aeroelastic Prediction Workshop," AIAA Paper 2015-0437, Jan. 2015.

${ }^{5}$ Silva, W., Chwalowski, P., and Perry, B., "Evaluation of linear, inviscid, viscous, and reduced-order modelling aeroelastic solutions of the AGARD 445.6 wing using root locus analysis," International Journal of Computational Fluid Dynamics, Vol. 28, Issue 3-4, 2014 , pages 122-139.

6"http://aiaa-dpw.larc.nasa.gov/".

7"'http://hiliftpw.larc.nasa.gov/".

8"http://fun3d.larc.nasa.gov/papers/FUn3D_Manual-12.7.pdf", NASA/TM-2015-218761.

${ }^{9}$ Pirzadeh, S. Z., "Advanced Unstructured Grid Generation for Complex Aerodynamic Applications," AIAA Paper 2008-7178, Aug. 2008.

${ }^{10}$ Samareh, J. A., "Unstructured Grids on NURBS Surfaces," AIAA Paper 1993-3454.

11 "http://nescacademy . nasa.gov/workshops/AePW2/public/".

12"'http://turbmodels. larc.nasa.gov/spalart.html".

${ }^{13}$ Spalart, P. R. and Allmaras, S. R., “A One-Equation Turbulence Model for Aerodynamic Flows,” La Recherche Aerospatiale, No. 1, 1994, pp 5-21.

${ }^{14}$ Venkatakrishnan, V., "Convergence to Steady State Solutions of the Euler Equations on Unstructured Grids with Limiter," Journal of Computational Physics, Vol. 118, No. 1, April 1995, pages 120-130.

${ }^{15}$ Roe, P. L., "Approximate Riemann Solvers, Parameter Vectors, and Difference Schemes," Journal of Computational Physics, Vol. 43, No. 2, October 1981, pages 357-372.

${ }^{16}$ Biedron, R. T. and Thomas, J. L., "Recent Enhancements to the FUN3D Flow Solver for Moving-Mesh Applications," AIAA Paper 20091360, Jan. 2009.

${ }^{17}$ Biedron, R. T. and Lee-Rausch, E. M., "Rotor Airloads Prediction Using Unstructured Meshes and Loose CFD/CSD Coupling," AIAA Paper 2008-7341, 2008.

${ }^{18}$ Batina, J. T., Seidel, D. A., Bland, S. R., and Bennet, R. M., "Unsteady Transonic Flow Calculations for Realistic Aircraft Configurations," AIAA Paper 1987-0850, 1987.

${ }^{19}$ Bartels, R. E., Rumsey, C. L., and Biedron, R. T., “CFL3D Version 6.4 - General Usage and Aeroelastic Analysis,” NASA TM 2006-214301, March 2006.

${ }^{20}$ MSC Software, Santa Ana, CA, MSC Nastran, 2008, http://www.mscsoftware.com/products/msc_nastran.cfm.

${ }^{21}$ Samareh, J. A., "Discrete Data Transfer Technique for Fluid-Structure Interaction,” AIAA Paper 2007-4309, June 2007.

${ }^{22}$ Vatsa, V. N., Carpenter, M. H., and Lockard, D. P., "Re-evaluation of an Optimized Second Order Backward Difference (BDF2OPT) Scheme for Unsteady Flow Applications,” AIAA Paper 2010-0122, Jan. 2010.

${ }^{23}$ Schuster, D. M., "Aerodynamic Measurements on a Large Splitter Plate for the NASA Langley Transonic Dynamics Tunnel," NASA TM 2001-210828, March 2001.
} 